\title{
Four-Body Co-Circular Central Configurations
}

\author{
Josep M. Cors* Gareth E. Roberts ${ }^{\dagger}$
}

October 25, 2011

\begin{abstract}
We classify the set of central configurations lying on a common circle in the Newtonian four-body problem. Using mutual distances as coordinates, we show that the set of four-body co-circular central configurations with positive masses is a two-dimensional surface, a graph over two of the exterior side-lengths. Two symmetric families, the kite and isosceles trapezoid, are investigated extensively. We also prove that a co-circular central configuration requires a specific ordering of the masses and find explicit bounds on the mutual distances. In contrast to the general four-body case, we show that if any two masses of a four-body co-circular central configuration are equal, then the configuration has a line of symmetry.
\end{abstract}

MSC Classifications: 70F10, 70F15, 37N05

Key Words: Celestial mechanics, central configurations, cyclic quadrilaterals, four-body problem

\section{Introduction}

The study of central configurations (c.c.'s) in the Newtonian $n$-body problem has become a vibrant sub-field of celestial mechanics. While much is known about specific cases, usually involving symmetry or assuming one or more bodies is infinitesimally small, there is less known about the general structure of the set of central configurations. For example, finiteness of c.c.'s (up to symmetry) was only recently established by Hampton and Moeckel [12] in the four-body problem, and by Albouy and Kaloshin [2] (except for masses in a codimension two subvariety) in the five-body problem.

In this work, we strive to deepen our understanding of the general set of central configurations by fully classifying the four-body co-circular central configurations (c.c.c.'s), that is, those four-body c.c.'s which also lie on a common circle (see Figure 1). According to Hampton [10], this problem was first posed by Alain Albouy. Requiring the bodies to lie on a common circle effectively reduces the problem of finding four-body c.c.'s by one dimension, allowing for a more substantial investigation. Although such a restriction may seem somewhat arbitrary, it is hoped that this work will prove

*Departament de Matemàtica Aplicada III, Universitat Politècnica de Catalunya, cors@epsem.upc.edu

$\dagger$ Dept. of Mathematics and Computer Science, College of the Holy Cross, groberts@radius.holycross.edu 


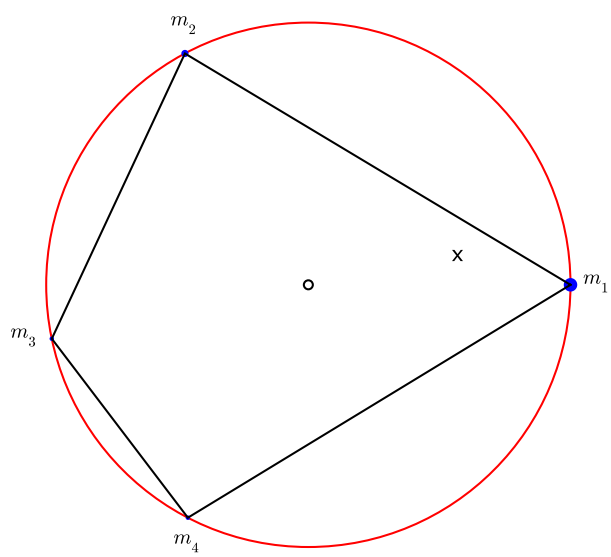

Figure 1: An example of a co-circular central configuration. The center of the circumscribing circle is marked with $\mathrm{a} \circ$ and the center of mass is denoted by $\mathrm{x}$.

useful in analyzing the general structure of four-body convex central configurations, that is, those c.c.'s forming a convex quadrilateral.

Co-circular c.c.'s are significant since they individually form the base of any spatial, pyramidal central configuration $[1,9]$. Such a three-dimensional configuration contains five bodies, with four co-planar bodies lying on a common circle and the fifth body (top of the pyramid) lying on the normal line through the center of the common circle. Albouy showed that the four co-planar bodies must be a c.c. of the planar four-body problem [1]. Therefore, our work also serves to classify the set of pyramidal central configurations.

Our approach uses only the mutual distances as coordinates. The fact that Ptolemy's theorem is so nicely expressed in these coordinates is critical to our results. Our main result is proving that the set of positions that yield a co-circular central configuration with positive masses is a two-dimensional surface, the graph of a differentiable function $f$ over two of the exterior sidelengths. The boundaries of this surface correspond to three important symmetric cases: a kite, an isosceles trapezoid, and a degenerate case where three bodies lie at the vertices of an equilateral triangle and the fourth body of the quadrilateral has zero mass. This last case corresponds to central configurations of the planar, circular, restricted four-body problem [13]. We also prove that for any c.c.c., the masses must be ordered in a precise fashion, with the largest body lying on the vertex between the two longest exterior sides, the smallest body opposite the largest, and the two largest (smallest, respectively) bodies lying on the longest (smallest, resp.) exterior side (see Section 4.2). In contrast to the famous result of MacMillan and Bartky [15] for convex 4-body c.c.'s, 
we demonstrate that not all choices of positive masses lead to a co-circular central configuration.

Moreover, we prove the somewhat surprising result that if just two masses of a four-body cocircular central configuration are equal, then the configuration is symmetric, either a kite or an isosceles trapezoid. These symmetric cases are explored in great detail in Section 3, in part to aid in the proof of our main result demonstrating the two-dimensional surface of c.c.c.'s. In Section 5.1, we prove sharp bounds for each exterior side-length, each diagonal, the ratio of the diagonals and the ratio of the second- and third-largest exterior side-lengths. We finish with some geometric results relating the positions of the bodies to the circumscribing circle.

Any planar central configuration, given the correct initial velocities, leads to a relative equilibrium solution in which the bodies rigidly rotate about their center of mass. If the c.c. is also co-circular, the circumcircle containing the bodies will also rotate about the center of mass. In [10], Hampton shows that only in the case of four equal masses positioned at the vertices of square, does the center of mass coincide with the center of the circumcircle, answering a question posed by Alain Chenciner. Some recent work in [18] proves the existence of a family of co-circular kite c.c.'s using Cartesian coordinates. A similar result also appears in [23], where Dziobek's equations are utilized, and the family is shown to exist for homogeneous force laws that include the Newtonian $n$-body problem as well as the $n$-vortex problem.

\section{Co-circular Central Configurations}

Suppose we have $n$ bodies with mass and position given by $m_{i}$ and $\mathbf{q}_{i} \in \mathbb{R}^{2}$ respectively, for $i=1, \ldots, n$. Denote $r_{i j}=\left\|\mathbf{q}_{i}-\mathbf{q}_{j}\right\|$ as the distance between the $i$ th and $j$ th bodies and let $\mathbf{q}=\left(\mathbf{q}_{1}, \ldots, \mathbf{q}_{n}\right) \in \mathbb{R}^{2 n}$. The center of mass of the system is $\mathbf{c}=M^{-1} \sum_{i=1}^{n} m_{i} \mathbf{q}_{i}$ where $M=$ $m_{1}+\cdots+m_{n}$ is the total mass. The equations of motion can be described by the Newtonian potential function

$$
U(\mathbf{q})=\sum_{i<j}^{n} \frac{m_{i} m_{j}}{r_{i j}}
$$

as simply

$$
m_{i} \ddot{\mathbf{q}}_{i}=\frac{\partial U}{\partial \mathbf{q}_{i}}, \quad i \in\{1,2, \ldots, n\} .
$$

Denote $I(\mathbf{q})$ as the moment of inertia,

$$
I(\mathbf{q})=\frac{1}{2} \sum_{i=1}^{n} m_{i}\left\|\mathbf{q}_{i}-\mathbf{c}\right\|^{2}=\frac{1}{2} \sum_{i=1}^{n} m_{i}\left\|\mathbf{q}_{i}\right\|^{2}-\frac{1}{2} M\|\mathbf{c}\|^{2}
$$

a characterization of the size of the system as measured from the center of mass.

A planar central configuration of the $n$-body problem is a configuration $\mathbf{x} \in \mathbb{R}^{2 n}$ which satisfies the algebraic equations

$$
\nabla U(\mathbf{x})+\lambda \nabla I(\mathbf{x})=0
$$

for some value of $\lambda$ (see [19], [20], [21], [26] or [29] for more details). Viewing $\lambda$ as a Lagrange multiplier, a central configuration is simply a critical point of $U$ subject to the constraint $I$ equals a constant. 
The $i$-th component in (1) is given by

$$
\sum_{j \neq i} \frac{m_{i} m_{j}\left(\mathbf{x}_{j}-\mathbf{x}_{i}\right)}{r_{i j}^{3}}+\lambda m_{i}\left(\mathbf{x}_{i}-\mathbf{c}\right)=0 .
$$

From this equation, it follows that if $\mathbf{x}=\left(\mathbf{x}_{1}, \mathbf{x}_{2}, \ldots, \mathbf{x}_{n}\right)$ is a planar central configuration, then

$$
\begin{aligned}
k \mathbf{x} & =\left(k \mathbf{x}_{1}, k \mathbf{x}_{2}, \ldots, k \mathbf{x}_{n}\right) \text { and } \\
R \mathbf{x} & =\left(R \mathbf{x}_{1}, R \mathbf{x}_{2}, \ldots, R \mathbf{x}_{n}\right)
\end{aligned}
$$

are also planar c.c.'s for any constant $k \neq 0$ and any $R \in S O(2)$. In the first case, the value of the multiplier $\lambda$ changes to $\lambda /|k|^{3}$ whereas the second case requires no change in $\lambda$. When counting central configurations it is standard to fix the size (a unique choice of $k$ ) and identify any configurations which are rotationally equivalent via the equivalence relation $\mathbf{x} \sim R \mathbf{x}$ for $R \in S O(2)$. Any planar central configuration leads to a two-parameter family of homographic solutions where each body travels along the same (scaled) solution of the Kepler problem.

\subsection{Mutual Distances}

In this section we derive the equations for a co-circular central configuration using the mutual distances $r_{i j}$ as coordinates. We make use of Ptolemy's theorem to give a derivation for c.c.c.'s that mirrors the approach used by Schmidt in [24].

Denote $\mathbf{r}=\left(r_{12}, r_{13}, r_{14}, r_{23}, r_{24}, r_{34}\right) \in \mathbb{R}^{+^{6}}$ as a vector of mutual distances. It is a straightforward calculation to check that

$$
I=\frac{1}{2 M} \sum_{i<j} m_{i} m_{j} r_{i j}^{2}
$$

For the case $n=4$, the mutual distances are not independent; they generically describe a tetrahedron in $\mathbb{R}^{3}$ rather than a configuration in the plane. Locating planar four-body central configurations requires an additional constraint found by setting the volume of the tetrahedron to zero. This constraint arises from the Cayley-Menger determinant

$$
V(\mathbf{r})=\left|\begin{array}{ccccc}
0 & 1 & 1 & 1 & 1 \\
1 & 0 & r_{12}^{2} & r_{13}^{2} & r_{14}^{2} \\
1 & r_{12}^{2} & 0 & r_{23}^{2} & r_{24}^{2} \\
1 & r_{13}^{2} & r_{23}^{2} & 0 & r_{34}^{2} \\
1 & r_{14}^{2} & r_{24}^{2} & r_{34}^{2} & 0
\end{array}\right|
$$

Assuming the $r_{i j}$ 's correspond to an actual tetrahedron, then the square of the volume of this tetrahedron is $V / 288$ [24]. However, it is important to note that $V$ may be positive for a given choice of 6 mutual distances, but that does not imply a configuration exists which realizes those particular distances. For instance, $\mathbf{r}=(2,4,1,7,4,1)$ gives $V(\mathbf{r})=3118$ but there is no physical configuration with these distances since $r_{12}=2, r_{13}=4, r_{23}=7$ does not satisfy the triangle inequality. Conversely, it is possible for all the triangle inequalities to be satisfied, yet have $V(\mathbf{r})<0$. One such example is the one parameter family $\mathbf{r}_{\mathbf{t}}=(1, t, 1,1,2 / t, 1)$ which gives $V\left(\mathbf{r}_{\mathbf{t}}\right)=-8 t^{-2}\left(t^{2}-\right.$ $2)^{2}$ which is strictly negative for all $t>0$ except at the unit square $t=\sqrt{2}$. 
To ensure a given vector $\mathbf{r}$ corresponds to an actual configuration of four bodies, either in the plane (with no three bodies collinear) or in space, it is necessary and sufficient that all strict triangle inequalities be satisfied and $V(\mathbf{r}) \geq 0$. Sufficiency follows from Theorem 9.7.3.4 and its proof in [6]. Specifically, let

$$
\mathcal{G}=\left\{\mathbf{r} \in \mathbb{R}^{+^{6}}: V(\mathbf{r}) \geq 0 \text { and } r_{i j}+r_{j k}>r_{i k} \text { for all triples of indices }(i, j, k) \text { where } i \neq j \neq k\right\} .
$$

We say that $\mathbf{r} \in \mathbb{R}^{+^{6}}$ is geometrically realizable if $\mathbf{r} \in \mathcal{G}$. Any geometrically realizable vector of mutual distances corresponds to a four-body planar, non-collinear configuration if $V(\mathbf{r})=0$ and a tetrahedron if $V(\mathbf{r})>0$.

We say that the bodies are ordered sequentially if they are numbered consecutively while traversing the boundary of the quadrilateral (either clockwise or counter-clockwise). For the remainder of this work, we will assume that any cyclic quadrilateral is ordered sequentially so that $r_{12}, r_{23}, r_{34}$ and $r_{14}$ are the lengths of the exterior sides of the quadrilateral and $r_{13}$ and $r_{24}$ are the lengths of the diagonals. Denote

$$
P(\mathbf{r})=r_{12} r_{34}+r_{14} r_{23}-r_{13} r_{24} .
$$

If a quadrilateral is ordered sequentially and is cyclic, then Ptolemy's theorem states that $P=0$. Moreover, for any convex quadrilateral ordered sequentially or for any tetrahedron, we have that $P \geq 0$, with equality if and only if the four bodies lie on a circle [5].

Let $\mathcal{P} \subset \mathcal{G}$ denote the set of all geometrically realizable $\mathbf{r}$ satisfying $P(\mathbf{r})=0$. The co-circular central configurations we seek to classify lie inside $\mathcal{P}$. We note that the codimension one level surfaces $\{V=0\}$ and $\{P=0\}$ meet tangentially at any point in $\mathcal{P}$. To prove this, we will show that $\nabla V$ and $\nabla P$ are parallel at any point in $\mathcal{P}$.

Let $\Delta_{i}$ be the oriented area of the triangle whose vertices contain all bodies except for the $i$-th body. For a quadrilateral ordered sequentially, we have $\Delta_{1}, \Delta_{3}>0$ and $\Delta_{2}, \Delta_{4}<0$. To find $\nabla V$ restricted to $\mathcal{P}$, we use an important formula concerning the Cayley-Menger determinant,

$$
\frac{\partial V}{\partial r_{i j}^{2}}=-32 \Delta_{i} \Delta_{j}
$$

This formula is only valid when restricting to planar configurations. The minus sign in equation (2) is not included in Dziobek's original paper [8] nor in several later works on four-body central configurations that utilize the Cayley-Menger determinant. However, checking equation (2) on the square configuration indicates the need for the minus sign. The correct formula appears in the doctoral thesis of Hampton [11] and has also been confirmed by Schmidt [25].

Let $\delta=r_{12} r_{13} r_{14} r_{23} r_{24} r_{34}$. Then, for any $\mathbf{r} \in \mathcal{P}$, we have

$$
\begin{aligned}
\frac{\partial V}{\partial r_{i j}}(\mathbf{r}) & =\frac{\partial V}{\partial r_{i j}^{2}} \cdot \frac{d\left(r_{i j}^{2}\right)}{d r_{i j}} \\
& =-64 r_{i j} \Delta_{i} \Delta_{j} \\
& =-64 r_{i j} \cdot \frac{ \pm 1}{16 r_{c}^{2}} \cdot r_{j k} r_{k l} r_{j l} \cdot r_{i k} r_{i l} r_{k l} \\
& =\frac{ \pm 4 \delta}{r_{c}^{2}} r_{k l}
\end{aligned}
$$


where $r_{c}$ is the circumradius of the cyclic quadrilateral and $k$ and $l$ are the two distinct indices in $\{1,2,3,4\}$ different from both $i$ and $j$. Here we have used the formula $\left|\Delta_{i}\right|=r_{j k} r_{k l} r_{j l} /\left(4 r_{c}\right)$ for the area of a triangle inscribed in a circle of radius $r_{c}$. Using the same ordering for indices as we used for the mutual distances, the signs of the partials of $V$ are $(+,-,+,+,-,+)$, which are identical to the signs of $\nabla P=\left(r_{34},-r_{24}, r_{23}, r_{14},-r_{13}, r_{12}\right)$. We have proven the following important lemma.

Lemma 2.1 For any $\mathbf{r} \in \mathcal{P}$,

$$
\nabla V(\mathbf{r})=\left(\frac{4}{r_{c}^{2}} \prod_{i<j} r_{i j}\right) \nabla P(\mathbf{r}) .
$$

In other words, on the the set of geometrically realizable vectors for which both $V$ and $P$ vanish, the gradients of these two functions are parallel.

We note that the above lemma does not hold in general and relies heavily on the fact that we are restricting to the family of cyclic quadrilaterals. Also, if the sign in equation (2) were not present, then $\nabla V(\mathbf{r})$ and $\nabla P(\mathbf{r})$ would point in opposite directions, violating the fact that any tetrahedron must have $P>0$. Lemma 2.1 shows that $\nabla V$ is superfluous when using the Lagrange multiplier method to locate co-circular central configurations. However, the CayleyMenger determinant is still required to ensure the configuration is geometrically realizable. For example, the family $\mathbf{r}_{\mathbf{t}}=(1, t, 1,1,2 / t, 1)$ mentioned earlier satisfies $V\left(\mathbf{r}_{\mathbf{t}}\right)<0$ (except for the unit square at $t=\sqrt{2}$ ), but also yields $P\left(\mathbf{r}_{\mathbf{t}}\right)=0$. Such a family needs to be excluded from the set of solutions.

Corollary 2.2 Assuming a sequential ordering, a four-body co-circular central configuration $\mathbf{r}$ is a critical point of the function

$$
U+\lambda M\left(I-I_{0}\right)+\sigma P
$$

satisfying $I=I_{0}, P=0$ and $V=0$.

The choice of $\lambda M$ as the first Lagrange multiplier in Corollary 2.2 simplifies the resulting equations. Using the six mutual distances as variables, we find

$$
\begin{array}{cl}
m_{1} m_{2}\left(r_{12}^{-3}-\lambda\right)=\sigma \frac{r_{34}}{r_{12}}, & m_{3} m_{4}\left(r_{34}^{-3}-\lambda\right)=\sigma \frac{r_{12}}{r_{34}} \\
m_{1} m_{3}\left(r_{13}^{-3}-\lambda\right)=-\sigma \frac{r_{24}}{r_{13}}, & m_{2} m_{4}\left(r_{24}^{-3}-\lambda\right)=-\sigma \frac{r_{13}}{r_{24}}, \\
m_{1} m_{4}\left(r_{14}^{-3}-\lambda\right)=\sigma \frac{r_{23}}{r_{14}}, & m_{2} m_{3}\left(r_{23}^{-3}-\lambda\right)=\sigma \frac{r_{14}}{r_{23}}
\end{array}
$$

Identical equations are obtained by using $V$ instead of $P$ in the function (3) and simplifying the resulting area formulas by assuming the bodies lie on a common circle. Following Schmidt's derivation of the equations for a c.c. of the planar four-body problem [24], we have grouped the 
above equations in pairs so that the product of the right-hand sides is simply $\sigma^{2}$. This yields the well-known relation of Dziobek [8]

$$
\left(r_{12}^{-3}-\lambda\right)\left(r_{34}^{-3}-\lambda\right)=\left(r_{13}^{-3}-\lambda\right)\left(r_{24}^{-3}-\lambda\right)=\left(r_{14}^{-3}-\lambda\right)\left(r_{23}^{-3}-\lambda\right)
$$

which is required of any planar 4-body c.c. (not just co-circular ones). Solving each of the three pairs of equations in (7) for $\lambda$ yields

$$
\lambda=\frac{r_{12}^{-3} r_{34}^{-3}-r_{13}^{-3} r_{24}^{-3}}{r_{12}^{-3}+r_{34}^{-3}-r_{13}^{-3}-r_{24}^{-3}}=\frac{r_{13}^{-3} r_{24}^{-3}-r_{14}^{-3} r_{23}^{-3}}{r_{13}^{-3}+r_{24}^{-3}-r_{14}^{-3}-r_{23}^{-3}}=\frac{r_{14}^{-3} r_{23}^{-3}-r_{12}^{-3} r_{34}^{-3}}{r_{14}^{-3}+r_{23}^{-3}-r_{12}^{-3}-r_{34}^{-3}} .
$$

If we set

$$
\begin{array}{ll}
s_{1}=r_{12}^{-3}+r_{34}^{-3}, & p_{1}=r_{12}^{-3} r_{34}^{-3}, \\
s_{2}=r_{13}^{-3}+r_{24}^{-3}, & p_{2}=r_{13}^{-3} r_{24}^{-3}, \\
s_{3}=r_{14}^{-3}+r_{23}^{-3}, & p_{3}=r_{14}^{-3} r_{23}^{-3},
\end{array}
$$

then equation (8) can be written as

$$
\lambda=\frac{p_{1}-p_{2}}{s_{1}-s_{2}}=\frac{p_{2}-p_{3}}{s_{2}-s_{3}}=\frac{p_{3}-p_{1}}{s_{3}-s_{1}}
$$

which means that $\left(s_{1}, p_{1}\right),\left(s_{2}, p_{2}\right),\left(s_{3}, p_{3}\right)$, viewed as points in $\mathbb{R}^{+2}$, must all lie on the same line with slope $\lambda$. This in turn, is equivalent to

$$
\left|\begin{array}{lll}
1 & 1 & 1 \\
s_{1} & s_{2} & s_{3} \\
p_{1} & p_{2} & p_{3}
\end{array}\right|=0
$$

a representation that leads to the particularly nice factorization

$$
\left(r_{13}^{3}-r_{12}^{3}\right)\left(r_{23}^{3}-r_{34}^{3}\right)\left(r_{24}^{3}-r_{14}^{3}\right)=\left(r_{12}^{3}-r_{14}^{3}\right)\left(r_{24}^{3}-r_{34}^{3}\right)\left(r_{13}^{3}-r_{23}^{3}\right) .
$$

Equation (9) is necessary and sufficient for a four-body planar central configuration given that the six mutual distances determine a geometrically realizable planar configuration. However, it does not ensure positivity of the masses. This equation can also be derived from the mass ratios given by Schmidt (equations (9) - (14)) in [24]. A similar and equivalent equation also appears on page 278 in the classic text of Wintner [29]. Note that equation (9) shows that if two sides with a common vertex are equal, then to be a c.c., there must be another pair of congruent sides sharing a common vertex. 


\subsection{Mass Ratios}

The ratios of the masses are easily obtained by dividing appropriate sets of equations from (4), (5) and (6). For example, we have

$$
\begin{aligned}
& \frac{m_{2}}{m_{1}}=\frac{\left(\lambda-r_{13}^{-3}\right) r_{13} r_{14}}{\left(r_{23}^{-3}-\lambda\right) r_{23} r_{24}}=\frac{\left(r_{14}^{-3}-\lambda\right) r_{13} r_{14}}{\left(\lambda-r_{24}^{-3}\right) r_{23} r_{24}}, \\
& \frac{m_{3}}{m_{1}}=\frac{\left(r_{12}^{-3}-\lambda\right) r_{12} r_{14}}{\left(r_{23}^{-3}-\lambda\right) r_{23} r_{34}}=\frac{\left(r_{14}^{-3}-\lambda\right) r_{12} r_{14}}{\left(r_{34}^{-3}-\lambda\right) r_{23} r_{34}}, \\
& \frac{m_{4}}{m_{1}}=\frac{\left(r_{12}^{-3}-\lambda\right) r_{12} r_{13}}{\left(\lambda-r_{24}^{-3}\right) r_{24} r_{34}}=\frac{\left(\lambda-r_{13}^{-3}\right) r_{12} r_{13}}{\left(r_{34}^{-3}-\lambda\right) r_{24} r_{34}}
\end{aligned}
$$

Requiring positive masses places additional constraints on the mutual distances. First, note that if $\lambda=r_{i j}^{-3}$ for any pair of indices $i, j$, then equations (4), (5) and (6) and nonzero masses imply that $\sigma=0$ and all $r_{i j}$ are equal (the regular tetrahedron solution). If $r_{13}^{-3}-\lambda<0$, then positivity of the masses implies $\sigma>0$ by (5). This in turn gives

$$
r_{12}, r_{14}, r_{23}, r_{34}<\lambda^{-1 / 3}<r_{13}, r_{24}
$$

If we had assumed that $r_{13}^{-3}-\lambda>0$, then the inequalities in (13) would be reversed. However, this is impossible, for then $P>r_{13} r_{24}>0$ and the configuration is not co-circular. Therefore, (13) is the only possibility and each side of the quadrilateral is shorter in length than either diagonal.

It can also be shown that the shortest exterior side must lie opposite the longest. This follows from

$$
\left(r_{12}^{-3}-\lambda\right)\left(r_{34}^{-3}-\lambda\right)=\left(r_{14}^{-3}-\lambda\right)\left(r_{23}^{-3}-\lambda\right)
$$

and inequalities (13). For example, suppose that $r_{12}$ is the largest exterior side-length. Then, since each factor in equation (14) is positive, we have $r_{12} \geq r_{14}$ implies $r_{23} \geq r_{34}$ and $r_{12} \geq r_{23}$ implies $r_{14} \geq r_{34}$. Together, these two implications show that $r_{34}$ is the shortest exterior side-length. The argument works exactly the same regardless of which exterior side is taken to be the longest. Without loss of generality, we label the bodies so that $r_{12}$ is the longest exterior side-length. Then, positivity of the masses implies

$$
r_{13}, r_{24}>r_{12} \geq r_{14}, r_{23} \geq r_{34}
$$

We can also assume that $r_{14} \geq r_{23}$ by an appropriate relabeling. More specifically, equations (4), (5) and (6) are invariant under the transformation $r_{13} \leftrightarrow r_{24}, r_{14} \leftrightarrow r_{23}, m_{1} \leftrightarrow m_{2}, m_{3} \leftrightarrow m_{4}$, which corresponds to interchanging bodies one and two, and bodies three and four. This interchange reverses the direction of the sequential ordering around the circle (clockwise to counterclockwise or vice versa). The choice $r_{14} \geq r_{23}$ imposes the relation $r_{13} \geq r_{24}$ between the two diagonals. To see this, note that for a cyclic quadrilateral ordered sequentially, the lengths of each diagonal $r_{13}$ and $r_{24}$ can be written as functions of the four exterior sides,

$$
r_{13}=\sqrt{\frac{a b}{c}} \text { and } r_{24}=\sqrt{\frac{a c}{b}},
$$


where $a=r_{12} r_{34}+r_{14} r_{23}, b=r_{12} r_{14}+r_{23} r_{34}$ and $c=r_{12} r_{23}+r_{14} r_{34}$. These can be derived using the Law of Cosines and the fact that opposite interior angles are supplementary [28]. If each equation in (15) holds, one can check that both $P=0$ and $V=0$ follows.

We then have

$$
\frac{r_{13}}{r_{24}}=\frac{b}{c}=\frac{r_{12} r_{14}+r_{23} r_{34}}{r_{12} r_{23}+r_{14} r_{34}}
$$

and $r_{13} \geq r_{24}$ if and only if $b-c \geq 0$ if and only if $\left(r_{14}-r_{23}\right)\left(r_{12}-r_{34}\right) \geq 0$. Since $r_{12}$ is the largest exterior side-length, $r_{14} \geq r_{23}$ if and only if $r_{13} \geq r_{24}$. Our final assumption is to fix the longest exterior side-length to one $\left(r_{12}=1\right)$, which specifies a particular choice of scaling. This is equivalent to holding the moment of inertia $I$ constant. Summarizing, without loss of generality, we can restrict to the set of mutual distances

$$
\Omega=\left\{\mathbf{r} \in \mathbb{R}^{+^{6}}: r_{13} \geq r_{24}>r_{12}=1 \geq r_{14} \geq r_{23} \geq r_{34}\right\} .
$$

Any co-circular central configuration with positive masses and our particular choice of labeling and scaling will correspond to some vector $\mathbf{r}$ of mutual distances in $\Omega$. A similar reduction was established by Hampton in [10].

Using the appropriate formula for $\lambda$ produces nice formulae for the mass ratios. For example, substituting $\lambda=\left(p_{2}-p_{3}\right) /\left(s_{2}-s_{3}\right)$ into the first equation in (10) yields, after some simplification,

$$
\frac{m_{2}}{m_{1}}=\frac{r_{23}^{2} r_{24}^{2}\left(r_{13}^{3}-r_{14}^{3}\right)}{r_{13}^{2} r_{14}^{2}\left(r_{24}^{3}-r_{23}^{3}\right)}
$$

Similar substitutions in (11) and (12) give, respectively,

$$
\frac{m_{3}}{m_{1}}=\frac{r_{23}^{2} r_{34}^{2}\left(r_{12}^{3}-r_{14}^{3}\right)}{r_{12}^{2} r_{14}^{2}\left(r_{23}^{3}-r_{34}^{3}\right)}
$$

and

$$
\frac{m_{4}}{m_{1}}=\frac{r_{24}^{2} r_{34}^{2}\left(r_{13}^{3}-r_{12}^{3}\right)}{r_{12}^{2} r_{13}^{2}\left(r_{24}^{3}-r_{34}^{3}\right)} .
$$

All of these ratios are positive and well-defined on $\Omega$, except for $m_{3} / m_{1}$ when $r_{12}=r_{14}$ and $r_{23}=r_{34}$ (a kite configuration). In this exceptional symmetric case, we use a different formula,

$$
\frac{m_{3}}{m_{1}}=\frac{\left(r_{12}^{-3}-\lambda\right) r_{12}^{2}}{\left(r_{23}^{-3}-\lambda\right) r_{23}^{2}}=\frac{\left(r_{13}^{3}-r_{12}^{3}\right)\left(r_{24}^{3}-r_{12}^{3}\right) r_{23}^{4}}{\left(r_{13}^{3}-r_{23}^{3}\right)\left(r_{24}^{3}-r_{23}^{3}\right) r_{12}^{4}}
$$

obtained by using $\lambda=\left(p_{1}-p_{2}\right) /\left(s_{1}-s_{2}\right)$. This can also be derived using equation (9).

Other mass ratios can be obtained in a similar fashion or by dividing pairs of equations from (16), (17) and (18). Since the masses can be scaled by any positive factor, we fix $m_{1}=1$ without any loss of generality. This choice will be made for the remainder of this work.

Summarizing the above, a configuration $\mathbf{r} \in \Omega$ with masses $m_{1}=1, m_{2}, m_{3}, m_{4}$ is a co-circular 
central configuration if and only if it is in the common zero set of the following six polynomials:

$$
\begin{aligned}
& F_{1}=r_{13}^{2}\left(r_{23}+r_{14} r_{34}\right)-\left(r_{14}^{2} r_{23}+r_{14} r_{34}\left(r_{23}^{2}+1\right)+r_{23} r_{34}^{2}\right), \\
& F_{2}=r_{24}^{2}\left(r_{14}+r_{23} r_{34}\right)-\left(r_{14}^{2} r_{23} r_{34}+r_{14}\left(r_{34}^{2}+r_{23}^{2}\right)+r_{23} r_{34}\right), \\
& F_{3}=\left(r_{13}^{3}-1\right)\left(r_{23}^{3}-r_{34}^{3}\right)\left(r_{24}^{3}-r_{14}^{3}\right)-\left(1-r_{14}^{3}\right)\left(r_{24}^{3}-r_{34}^{3}\right)\left(r_{13}^{3}-r_{23}^{3}\right), \\
& F_{4}=m_{2} r_{13}^{2} r_{14}^{2}\left(r_{24}^{3}-r_{23}^{3}\right)-r_{23}^{2} r_{24}^{2}\left(r_{13}^{3}-r_{14}^{3}\right), \\
& F_{5}=m_{3} r_{14}^{2}\left(r_{23}^{3}-r_{34}^{3}\right)-r_{23}^{2} r_{34}^{2}\left(1-r_{14}^{3}\right), \\
& F_{6}=m_{4} r_{13}^{2}\left(r_{24}^{3}-r_{34}^{3}\right)-r_{24}^{2} r_{34}^{2}\left(r_{13}^{3}-1\right) .
\end{aligned}
$$

We will refer to this system of six equations as System I. The polynomials $F_{1}$ and $F_{2}$ are easily obtained from the two equations for $r_{13}$ and $r_{24}$ given in (15). $F_{3}$ is a restatement of equation (9) and the remaining three polynomials are easily derived from equations (16), (17) and (18) respectively.

There are two ways of approaching the problem of classifying co-circular c.c.'s. First, given three positive masses $m_{2}, m_{3}$ and $m_{4}$, one can try to simultaneously solve System I. Since this is six equations in five unknowns, there is typically no solution. On the other hand, there is the inverse approach: For what set of mutual distances does there correspond a set of positive masses that make the configuration central? This is the approach pursued here. It involves a simpler analysis, restricting to the set of solutions to $\left\{F_{1}=0, F_{2}=0, F_{3}=0\right\}$ lying in $\Omega$.

\section{Symmetric Cases}

Before studying the full set of co-circular central configurations, we analyze the two symmetric cases: a kite configuration and an isosceles trapezoid. These symmetric cases occur on the boundaries of the space $\Omega$, sharing a common point corresponding to the square central configuration with equal masses. There is a one-parameter family for each case.

\subsection{Kite Configurations}

We call a convex quadrilateral a kite configuration if two opposite bodies lie on an axis of symmetry of the configuration (see Figure 2). Using the bounds that define $\Omega$, it is straight-forward to show that a co-circular c.c. is a kite if and only if one pair of opposite bodies lie on a diameter of the circumscribing circle. To see that any kite configuration is automatically a central configuration, note that equation (9) is immediately satisfied when $r_{12}=r_{14}=1$ and $r_{23}=r_{34}$. This gives a kite central configuration with an axis of symmetry between bodies 1 and 3. A similar kite exists with symmetry axis between bodies 2 and 4 , but is excluded here by our choice that $r_{14} \geq r_{23}$.

We set $r_{23}=r_{34}=x$ and the diagonals $r_{13}=c$ and $r_{24}=2 x / c$ where $x \in(0,1]$ is a parameter and $c=\sqrt{1+x^{2}}$. These distances, together with $r_{12}=r_{14}=1$, describe a planar $(V=0)$, kite central configuration that also satisfies Ptolemy's relation $P=0$. It only remains to check whether the mass ratios are positive. First note that under these assumptions, $m_{2}=m_{4}$, a result we expect from symmetry. Expressions (16), (18) and (19) with $m_{1}=1$ give $m_{2}=m_{4}=m$ and $m_{3}=\alpha m$, where

$$
m=\frac{4 x\left(c^{3}-1\right)}{c\left(8-c^{3}\right)} \quad \text { and } \quad \alpha=\frac{c\left(8 x^{3}-c^{3}\right)}{4\left(c^{3}-x^{3}\right)} .
$$




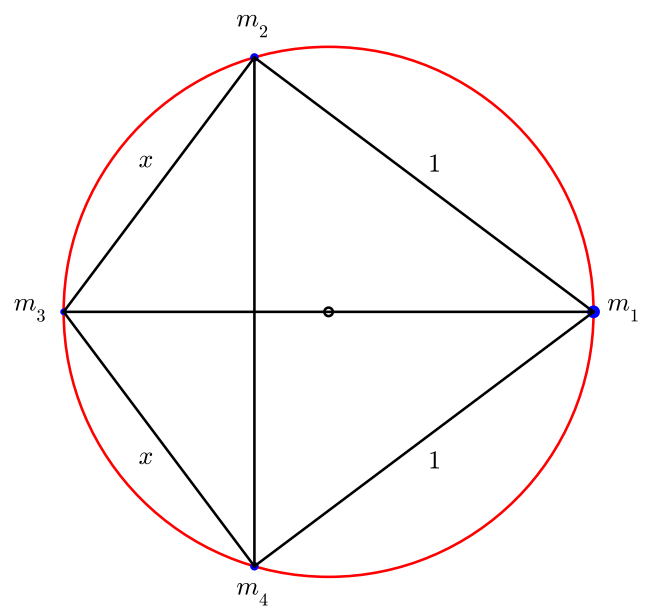

Figure 2: An example of a co-circular kite central configuration with $m_{2}=m_{4}$. The center of the circumscribing circle is marked with a $\circ$.

The value for $m_{2}=m_{4}$ is positive since $1<c \leq \sqrt{2}$. For $x \in(0,1], \alpha$, and consequently $m_{3}$, is positive if and only if $1 / \sqrt{3}<x \leq 1$. We have $m_{3}=0$ and $r_{24}=1$ when $x=1 / \sqrt{3}$. In this degenerate case, bodies 1, 2 and 4 form an equilateral triangle and the configuration is a c.c. of the planar, circular, restricted four-body problem. At the other endpoint, setting $x=1$ yields a square and all masses equal to one, as expected. Figure 3 displays the values of the masses as a function of the parameter $x$.

Theorem 3.1 There exists a one-parameter family of co-circular kite central configurations with bodies one and three lying on the diameter of the circumscribing circle. The masses are $m_{1}=$ $1, m_{2}=m_{4}=m$ and $m_{3}=\alpha m$ and are ordered $m_{1} \geq m_{2}=m_{4} \geq m_{3}$ with equality if and only if the configuration is a square. At one end of the family, $x=1 / \sqrt{3}$, is a c.c. of the planar, circular, restricted four-body problem, with bodies 1, 2 and 4 forming an equilateral triangle and $m_{3}=0$. At the other end, $x=1$, is the square with equal masses.

Proof: The only item remaining to show is the ordering of the masses, which is clear from Figure 3, but can be shown rigorously with straight-forward analysis. We first show $m_{3} \leq m_{2}$ by verifying that $\alpha \leq 1$. On the interval $1 / \sqrt{3} \leq x \leq 1$, this is equivalent to showing $c\left(8 x^{3}-c^{3}\right) \leq 4\left(c^{3}-x^{3}\right)$ or

$$
c\left(8 x^{3}-4 x^{2}-4\right) \leq\left(x^{2}+1\right)^{2}-4 x^{3} .
$$

This inequality becomes an equality at $x=1$. Assuming $x<1$, we can divide both sides by the common positive factor $1-x$ to obtain

$$
-4 c\left(2 x^{2}+x+1\right)<4 x+(1-x)^{3}
$$




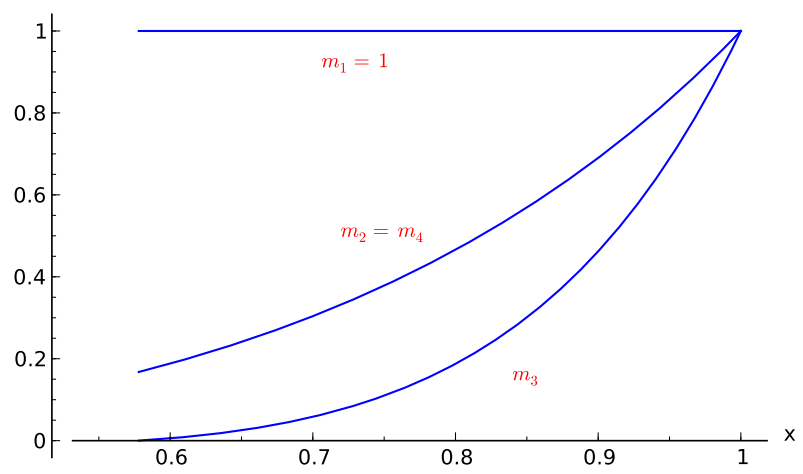

Figure 3: The values of the masses for the co-circular kite configurations.

which is clearly valid for $1 / \sqrt{3} \leq x<1$.

Next, we show that $m \leq 1$ for $1 / \sqrt{3} \leq x \leq 1$. This is equivalent to $4 x\left(c^{3}-1\right) \leq c\left(8-c^{3}\right)$ or

$$
\left(x^{2}+1\right)^{2}-4 x \leq 4 c\left(2-x\left(x^{2}+1\right)\right) .
$$

This inequality also becomes an equality at $x=1$. As before, assuming $x<1$, we can divide both sides by the common positive factor $1-x$ to obtain

$$
-\left(x^{3}+x^{2}+3 x-1\right)<4 c\left(x^{2}+x+2\right) .
$$

It is straight-forward to show that the polynomial on the left-hand side of this last inequality is negative for $1 / \sqrt{3} \leq x<1$ while the right-hand side is clearly positive. The above arguments also show that the relationships between the masses are strict inequalities if $x<1$. This completes the proof.

\section{Remarks:}

1. While our work was being completed, a similar theorem appeared in both [18] and [23].

2. It can be shown with straight-forward calculus that both $m$ and $\alpha$ are strictly increasing functions of $x$ for $1 / \sqrt{3} \leq x \leq 1$. Thus, $m_{2}, m_{3}$ and $m_{4}$ are strictly increasing functions of the common side-length $r_{23}=r_{34}$.

3. The center of mass for the kite lies closest to the largest body $\left(m_{1}\right)$ for $x<1$ and is equivalent to the circumcenter at $x=1$. If the circumcenter is taken to be the origin, and $m_{1}$ is positioned on the positive $x$-axis, then a short calculation shows that the center of mass for $x=1 / \sqrt{3}$ is $((41 \sqrt{3}-21) / 104,0) \approx(0.4809,0)$. By the main result of Hampton [10] and by continuity, the center of mass has coordinates $(\bar{x}, 0)$ with $\bar{x}>0$ for $1 / \sqrt{3} \leq x<1$. Moreover, a graph of the first coordinate of the center of mass shows that it is a strictly decreasing function of $x$.

Denote $\theta_{i j}$ as the arc along the cirmcumscribing circle between bodies $i$ and $j$. Since we have specified $r_{12}=1$ as the longest exterior side, $\theta_{12}$ will be the largest arc between any two consecutive 
bodies. For the kite family, the circumradius $r$ is simply $r=c / 2$ and $\theta_{12}$ can be expressed in terms of the parameter $x$ as $\theta_{12}=2 \arctan (1 / x)$, a monotonically decreasing function. The maximum value of $\theta_{12}$ is $120^{\circ}\left(x=1 / \sqrt{3}, m_{3}=0\right)$ while the minimum value is $90^{\circ}(x=1$, square $)$. For the smallest arc between any two consecutive bodies, we have $\theta_{23}=\theta_{34}=2 \arctan (x)$, an increasing function of $x$ ranging from $60^{\circ}$ to $90^{\circ}$ for $1 / \sqrt{3}<x \leq 1$. Note also that the maximum interior angle $\angle_{234}$ of the quadrilateral is equivalent to $\theta_{12}$ while the minimum interior angle $\angle_{214}$ is equivalent to $\theta_{23}$. Therefore, the largest interior angle of a co-circular kite c.c. has a supremum of $120^{\circ}\left(m_{3}=0\right)$ and a minimum of $90^{\circ}$ (square). Furthermore, the arc length along the circumscribing circle between bodies 1 and 2 is given by

$$
\operatorname{Arc}(x)=\theta_{12} \cdot \frac{c}{2}=\sqrt{x^{2}+1} \arctan \left(\frac{1}{x}\right) .
$$

From this formula, it is clear that $\operatorname{Arc}(x)$ is a decreasing function, with a supremum of $2 \pi /(3 \sqrt{3}) \approx$ $1.2092\left(m_{3}=0\right)$ and a minimum attained at the square configuration of $2^{-3 / 2} \pi \approx 1.1107$.

\subsection{The Isosceles Trapezoid}

In this section we verify the existence of a one-parameter family of isosceles trapezoids. Some of the results depend on symbolic calculations where Maple [16] is used to compute Gröbner bases and/or Sturm sequences. A Maple worksheet containing all the pertinent computations is available at http://mathcs.holycross.edu/ groberts/Papers/papers.html .

Any isosceles trapezoid, that is, a trapezoid whose legs are congruent, can be circumscribed in a circle with center lying on the axis of symmetry. In our setup, the isosceles trapezoids correspond to the case where $r_{14}=r_{23}$ and $r_{13}=r_{24}$. Equation (16) then implies that $m_{1}=m_{2}$. From equations (17), (18) and (9), we have

$$
\frac{m_{3}}{m_{4}}=\frac{r_{13}^{2} r_{23}^{2}\left(r_{12}^{3}-r_{14}^{3}\right)\left(r_{24}^{3}-r_{34}^{3}\right)}{r_{14}^{2} r_{24}^{2}\left(r_{23}^{3}-r_{34}^{3}\right)\left(r_{13}^{3}-r_{12}^{3}\right)}=\frac{r_{13}^{2} r_{23}^{2}\left(r_{24}^{3}-r_{14}^{3}\right)}{r_{14}^{2} r_{24}^{2}\left(r_{13}^{3}-r_{23}^{3}\right)},
$$

so that $m_{3}=m_{4}$ follows as well. The equality of the two pairs of masses is expected from the symmetry of the configuration. This fact appears in the well-known paper of MacMillan and Bartky [15]. It also turns out that if the c.c. is assumed to be co-circular, then equality of two pairs of adjacent masses implies the configuration is an isosceles trapezoid. It is unknown if the same fact holds for general four-body convex central configurations.

Lemma 3.2 If $m_{1}=m_{2}$ and $m_{3}=m_{4}$, then the corresponding co-circular central configuration must be an isosceles trapezoid.

Proof: If $m_{1}=m_{2}$ and $m_{3}=m_{4}$, then equations (16) and (20) imply

$$
\begin{aligned}
& r_{23}^{2} r_{24}^{2}\left(r_{13}^{3}-r_{14}^{3}\right)-r_{13}^{2} r_{14}^{2}\left(r_{24}^{3}-r_{23}^{3}\right)=0, \\
& r_{13}^{2} r_{23}^{2}\left(r_{24}^{3}-r_{14}^{3}\right)-r_{14}^{2} r_{24}^{2}\left(r_{13}^{3}-r_{23}^{3}\right)=0 .
\end{aligned}
$$

Taking the difference of these two equations and factoring the result yields

$$
\left(r_{13}-r_{24}\right)\left(r_{13}^{2} r_{14}^{2} r_{24}^{2}+r_{13}^{2} r_{23}^{2} r_{24}^{2}+r_{13} r_{14}^{3} r_{23}^{2}+r_{13} r_{14}^{2} r_{23}^{3}+r_{14}^{3} r_{23}^{2} r_{24}+r_{14}^{2} r_{23}^{3} r_{24}\right)=0,
$$




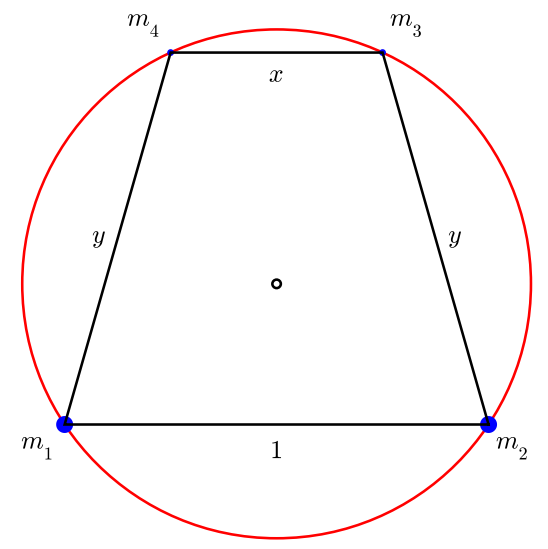

Figure 4: An example of an isosceles trapezoid central configuration. The center of the circumscribing circle is marked with a $\circ$.

which implies $r_{13}=r_{24}$ since the mutual distances are real, positive numbers. Since the configuration is on a circle, it follows that $r_{14}=r_{23}$, and the configuration is an isosceles trapezoid.

Remark: A slightly stronger result exists. In fact, for co-circular c.c.'s, only one pair of adjacent masses need be equal (e.g., $m_{1}=m_{2}$ or $m_{3}=m_{4}$ ) to imply the configuration is an isosceles trapezoid (see Corollary 4.7).

To describe the family of isosceles trapezoid central configurations, we set $r_{12}=1, r_{34}=x, r_{14}=$ $r_{23}=y$ where $0 \leq x \leq 1$ and $x \leq y \leq 1$ are dependent parameters (see Figure 4). Using Ptolemy's theorem, we have $r_{13}=r_{24}=\sqrt{x+y^{2}}$. One can then check that $V=0$ is satisfied for this family. As before, we fix $m_{1}=1$ so that $m_{2}=1$. Then, by equations (17) and (20), $m_{3}=m_{4}=m$, where

$$
m=\frac{x^{2}\left(1-y^{3}\right)}{y^{3}-x^{3}}
$$

In order for the trapezoid to be a c.c., equation (9) must also be satisfied. Unlike the kite family, this yields a non-trivial condition. Letting

$$
T(x, y)=\left(y^{2}+x\right)^{3 / 2}\left(2 y^{3}-x^{3}-1\right)-y^{3}-x^{3} y^{3}+2 x^{3},
$$

the constraint $T=0$ must be satisfied in order for the isosceles trapezoid to be a central configuration.

A graph of the level curve $T=0$ in the $x y$-plane for $0 \leq x \leq 1, x \leq y \leq 1$ is shown in Figure 5. Note that while the smallest side of the trapezoid (parallel to the base) can range 


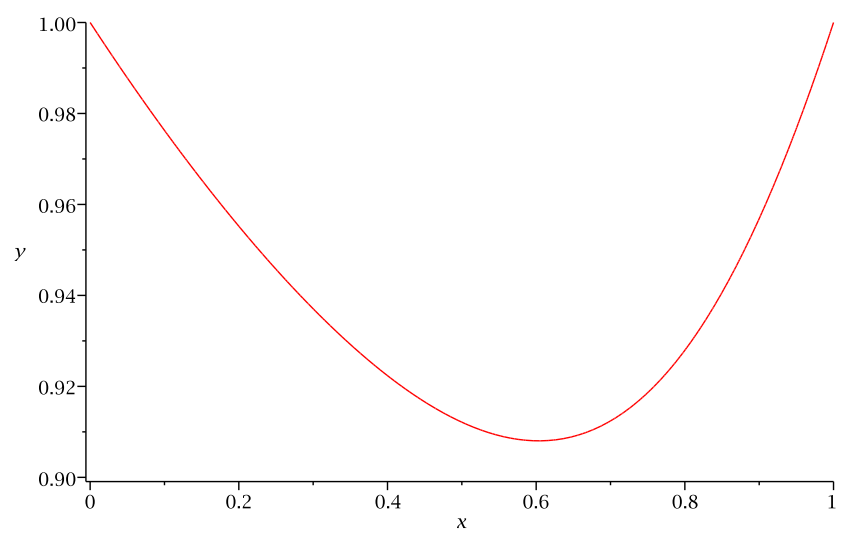

Figure 5: The relationship between the two distances $x=r_{34}$ and $y=r_{14}=r_{23}$ in the isosceles trapezoid family of central configurations.

from 0 to 1 , the length of the congruent legs is considerably constrained between approximately 0.908 and 1. At the point $(0,1)$ (left endpoint of the curve), we have an equilateral triangle $\left(r_{34}=0, r_{23}=r_{24}=r_{14}=r_{12}=1\right)$ and $m_{3}=m_{4}=0$, corresponding to an equilibrium point of the planar, circular, restricted, three-body problem where two infinitesimal masses are located at the same Lagrange point. At the point $(1,1)$ (right endpoint), we have the equal mass square central configuration.

Next we show that $T(x, y)=0$ implicitly defines a differentiable function $y=\tau(x)$ for $x \in$ $[0,1]$. Consequently, there exists a one-parameter family of co-circular isosceles trapezoid central configurations parametrized by $r_{34}=x$. The following Lemma and its proof will be important in the verification of Theorem 4.3, establishing the surface of co-circular c.c.'s.

Lemma 3.3 For each value of $x \in(0,1]$, there exists a unique value of $y \in[x, 1]$ such that $T(x, y)=$ 0 . Moreover, the distance parameter $y$ can be written as a differentiable function, $y=\tau(x)$.

Proof: Since $r_{13}=r_{24}>1$ on $\Omega$, we have that $\sqrt{x+y^{2}}>1$ or $y>\sqrt{1-x}$. Since $1 \geq r_{23} \geq r_{34}$ on $\Omega$, we also have that $x \leq y \leq 1$. Thus we can restrict to the subset $\Lambda$ of the $x y$-plane defined as

$$
\Lambda=\left\{(x, y) \in \mathbb{R}^{2}: 0 \leq x \leq 1, x \leq y \leq 1 \text { and } y \geq \sqrt{1-x}\right\} .
$$

For $x \geq 0$, the curves $y=\sqrt{1-x}$ and $y=x$ intersect at $x=(-1+\sqrt{5}) / 2$. Thus, on $\Lambda$, $y \geq(-1+\sqrt{5}) / 2$.

Note that $T(x, 1)=\left(1-x^{3}\right)\left((1+x)^{3 / 2}-1\right) \geq 0$ for $0 \leq x \leq 1$ with equality only at $x=0$ or $x=1$. Furthermore,

$$
\begin{aligned}
T(x, \sqrt{1-x}) & =\left(1-x^{3}\right)\left((1-x)^{3 / 2}-1\right), \\
T(x, x) & =\left(1-x^{3}\right)\left(x^{3}-\left(x^{2}+x\right)^{3 / 2}\right),
\end{aligned}
$$

each of which are negative for $0<x<1$. Fix an $x \in(0,1)$ and consider $T(x, y)=T_{x}(y)$ as a function in the variable $y$. It follows that $T_{x}<0$ at the lower boundary of $\Lambda$ and $T_{x}>0$ at the 
upper boundary of $\Lambda$. Since $T$ is continuous, there is a solution to $T(x, y)=0$ inside $\Lambda$ for each $x \in(0,1)$. To see that it is unique, we compute that

$$
\frac{\partial^{2} T}{\partial y^{2}}=3\left[-2 y\left(x^{3}+1\right)+\sqrt{x+y^{2}}\left(16 y^{3}+4 x y-x^{3}-1\right)+\frac{y^{2}\left(4 y^{3}+2 x y-x^{3}-1\right)}{\sqrt{x+y^{2}}}\right] .
$$

The quantity $4 y^{3}+2 x y-x^{3}-1$ is strictly positive on $\Lambda$. To see this, note that the inequality $x+y^{2}>1$ implies that $x+2 y^{2}>1+y^{2}$. Then, we have

$$
\begin{aligned}
4 y^{3}+2 x y-x^{3}-1 & =2 y\left(x+2 y^{2}\right)-x^{3}-1 \\
& >2 y\left(1+y^{2}\right)-x^{3}-1 \\
& \geq 2 y\left(1+y^{2}\right)-y^{3}-1 \quad(\text { since } x \leq y) \\
& =y^{3}+2 y-1 \\
& \geq \frac{(-1+\sqrt{5})^{3}}{8}+\sqrt{5}-2 \quad(\text { since } y \geq(-1+\sqrt{5}) / 2) \\
& >0 .
\end{aligned}
$$

Next, using the inequalities $\sqrt{x+y^{2}}>1$ and $x \leq 1$, we have

$$
\begin{aligned}
\frac{\partial^{2} T}{\partial y^{2}} & >3\left[-2 y\left(x^{3}+1\right)+\left(x+y^{2}\right)^{1 / 2}\left(16 y^{3}+4 x y-x^{3}-1\right)\right] \\
& >3\left[-2 y\left(x^{3}+1\right)+16 y^{3}+4 x y-x^{3}-1\right] \\
& =3\left[4 y\left(4 y^{2}+x\right)-(2 y+1)\left(x^{3}+1\right)\right] \\
& >3\left[4 y\left(1+3 y^{2}\right)-2(2 y+1)\right] \\
& =6\left(6 y^{3}-1\right) \\
& >0 \quad(\text { since } y \geq(-1+\sqrt{5}) / 2>\sqrt[3]{1 / 6}) .
\end{aligned}
$$

This proves that $\frac{\partial^{2} T}{\partial y^{2}}>0$ on $\Lambda$. Then, since $T_{x}<0$ on the lower boundary of $\Lambda$, and $T_{x}>0$ on the upper boundary of $\Lambda$, it follows that

$$
\left.\frac{d}{d y}\left(T_{x}(y)\right)\right|_{y=\hat{y}}>0
$$

where $\hat{y}$ satisfies $T_{x}(y)=0$ and is the smallest such $y$-value to satisfy this equation. Since $\frac{\partial^{2} T}{\partial y^{2}}>0$, $y=\hat{y}$ is the unique solution to $T_{x}(y)=0$. This proves there exists a function $y=\tau(x)$ for $0<x<1$ satisfying $T(x, \tau(x))=0$ on $\Lambda$.

Finally, since $T(1,1)=0$, set $\tau(1)=1$. We compute that $\frac{\partial T}{\partial y}(1,1)=6(2 \sqrt{2}-1)>0$. Applying the implicit function theorem, this fact and inequality (22) proves that $y=\tau(x)$ is a differentiable function of $x$ for $0<x \leq 1$.

\section{Remarks:}


1. For $x=1$ (the square), we have $\tau^{\prime}(1)=1 / 2$ by implicit differentiation. Although $x=r_{34}=0$ is not a true physical solution, we can extend $\tau(x)$ to the closed interval $[0,1]$ by defining $\tau(0)=1$. Another computation gives $T(0,1)=0, \frac{\partial T}{\partial y}(0,1)=6>0$ and $\tau^{\prime}(0)=-1 / 4$.

2. It can be shown that $0.9<\tau(x)<1$ for $0<x<1$. The fact that $\tau(x)<1$ for $0<x<1$ follows quickly from $T(x, 1)=\left(1-x^{3}\right)\left((1+x)^{3 / 2}-1\right)$. To show that $\tau(x)>0.9$, we introduce the new variable $z=\sqrt{x+y^{2}}$ and use Maple to compute a Gröbner basis for the ideal generated by the two polynomials $T(x, y=9 / 10)$ and $x+(9 / 10)^{2}-z^{2}[7]$. This produces a polynomial in $z$ of degree 9 which, using Sturm's theorem [27], contains no real roots between 0 and 2. Consequently, since $\tau(x)$ is a continuous function and $\tau(0)=\tau(1)=1$, it follows that $\tau(x)>0.9$. While this is a computer-assisted argument, it only depends on symbolic and exact integer computations.

3. The minimum of $\tau(x)$ can be found by simultaneously solving the equations $T(x, y)=0$ and $\frac{\partial T}{\partial x}=0$ on $\Lambda$. As above, introducing the variable $z=\sqrt{x+y^{2}}$, we use Maple to compute a Gröbner basis of the relevant ideal. Numerically approximating the roots of two onevariable polynomials yields a unique minimum at $x=0.6035381491, y=0.9080259298$. This calculation was also confirmed using the free software Sage [22] and agrees with the data given in Table II in [15].

In [15], MacMillan and Bartky show that a unique isosceles trapezoid c.c. exists for any choice of masses $m_{1}=m_{2}$ and $m_{3}=m_{4}$. They use a different parametrization than ours, setting $r_{23}=$ $\kappa \sqrt{r_{12} r_{34}}$, with $\kappa$ as a parameter. In our notation, $\kappa=y / \sqrt{x}$. The same result is proven by Albouy in [3]. For completeness sake, we provide another proof using symbolic computation with Maple.

Theorem 3.4 The mass parameter $m$ is an increasing function of $x$ satisfying $m(0)=0$ and $m(1)=1$. Consequently, for each choice of the mass parameter $m$, there exists a unique isosceles trapezoid central configuration. Moreover, the largest pair of equal masses in the isosceles trapezoid central configuration lie on the larger base.

Proof: By Lemma 3.3 and its proof, there exists a differentiable function $\tau(x)$ for $x \in[0,1]$ satisfying $T(x, y=\tau(x))=0$. Moreover, $x<\tau(x)<1$ for $x \in[0,1)$ and $\tau(1)=1$. Thus, the mass parameter $m$ is non-negative on $x \in[0,1]$ and is given by

$$
m(x)=\left\{\begin{array}{cc}
\frac{x^{2}\left(1-(\tau(x))^{3}\right)}{(\tau(x))^{3}-x^{3}} & \text { if } 0 \leq x<1 \\
1 & \text { if } x=1
\end{array}\right.
$$

Using $\tau(1)=1$ and $\tau^{\prime}(1)=1 / 2$, a straight-forward application of L'Hôpital's rule gives

$$
\lim _{x \rightarrow 1^{-}} m(x)=1
$$

so that $m(x)$ is a continuous function on $[0,1]$. We compute that, for $x \in[0,1)$,

$$
\frac{d m}{d x}=\frac{x\left[\left(1-(\tau(x))^{3}\right)\left(2(\tau(x))^{3}+x^{3}\right)-3 x(\tau(x))^{2}\left(1-x^{3}\right) \tau^{\prime}(x)\right]}{\left((\tau(x))^{3}-x^{3}\right)^{2}}
$$


We claim that this quantity is positive for $0<x<1$. Note that since $\tau(0)=1$ and $\tau(x)<1$ for $x<1$, we have that $\tau^{\prime}(x)<0$ for $x$ near 0 . Consequently, $d m / d x>0$ for $x$ sufficiently close to 0 . To show that $d m / d x>0$ for all $x \in(0,1)$, we use Gröbner bases and Sturm's theorem [27]. Using the fact that $\tau^{\prime}(x)=-\frac{\partial T}{\partial x} / \frac{\partial T}{\partial y}$ and inequality (22), it is sufficient to show that

$$
m_{x}=\left(1-y^{3}\right)\left(2 y^{3}+x^{3}\right) \frac{\partial T}{\partial y}+3 x y^{2}\left(1-x^{3}\right) \frac{\partial T}{\partial x}
$$

is strictly positive on the curve $y=\tau(x)$ for $x \in(0,1)$. As with previous arguments, introduce the variable $z=\sqrt{x+y^{2}}$. Computing a Gröbner basis with respect to the lexicographic ordering $z>y>x$ for the ideal generated by

$$
\begin{aligned}
T & =z^{3}\left(2 y^{3}-x^{3}-1\right)-y^{3}-x^{3} y^{3}+2 x^{3}, \\
m_{x} & =\left(1-y^{3}\right)\left(2 y^{3}+x^{3}\right) \frac{\partial T}{\partial y}+3 x y^{2}\left(1-x^{3}\right) \frac{\partial T}{\partial x}, \\
Z & =x+y^{2}-z^{2},
\end{aligned}
$$

yields a polynomial in $x$ of the form

$$
x^{4}(x-1)^{2}\left(x^{2}+x+1\right)^{2} \cdot \phi(x)
$$

where $\phi(x)$ is degree 48. Using the Maple commands sturmseq and sturm, we apply Sturm's theorem to conclude that $\phi(x)$ has no real roots in $[0,1]$. Consequently, the function $m_{x}$ does not change sign along the curve $y=\tau(x)$. Since $m_{x}>0$ for $x$ sufficiently close to 0 , it follows that $m_{x}>0$ on the curve $y=\tau(x)$ for all $x \in(0,1)$.

Next, consider the arc $\theta_{12}$ along the cirmcumscribing circle between bodies 1 and 2 . We have $\theta_{12}=2 \arcsin \left(\frac{1}{2 r_{c}}\right)$ where $r_{c}$ is the circumradius

$$
r_{c}=y \sqrt{\frac{x+y^{2}}{4 y^{2}-(1-x)^{2}}} .
$$

At $(0,1)$ (degenerate equilateral triangle), we have $r_{c}=1 / \sqrt{3}$ and at $(1,1)$ (square), we have $r_{c}=\sqrt{2} / 2$, as expected. The maximum value of $\theta_{12}$ will occur at the minimum value of the circumradius. We consider the quantity $r_{c}^{2}$ and claim that $\frac{d r_{c}^{2}}{d x}$ is positive on $0<x \leq 1$. This is equivalent to showing that

$$
y\left(2 y^{2}+2 x y^{2}+x^{2}-1\right) \frac{\partial T}{\partial y}-\left(8 y^{4}-2(1-x)^{2}\left(x+2 y^{2}\right)\right) \frac{\partial T}{\partial x}
$$

is strictly positive on $0<x \leq 1$. This is verified with Maple using the same symbolic computing techniques as in the proof of Theorem 3.4. In this case, we obtain a polynomial in $y$ which, by Sturm's theorem, has no roots between 0.9 and 1 . This means that expression (23) does not change sign along the curve $y=\tau(x)$. Since expression (23) evaluates to $48(2 \sqrt{2}-1)>0$ at the square $x=1, y=1$, it must be positive along all of $y=\tau(x)$. Thus, the infimum of the circumradius is $r=1 / \sqrt{3}$ and $\theta_{12}$ is a strictly decreasing function of $x$. 
Next, consider the $\operatorname{arc} \theta_{14}$ between bodies 1 and 4 . In this case, we have $\theta_{14}=2 \arcsin \left(\frac{y}{2 r_{c}}\right)$. A similar argument as above, using Maple, shows that the quantity $\frac{y}{2 r_{c}}$ is a strictly decreasing function for $0 \leq x \leq 1$. Consequently, $\theta_{14}$ is also a strictly decreasing function of $x$. This shows that the largest interior angle, $\angle_{234}$, given by $\left(\theta_{12}+\theta_{14}\right) / 2$, is a strictly decreasing function of $x$. We have given a computer-assisted proof of the following theorem.

Theorem 3.5 The largest arc $\theta_{12}$ in the isosceles trapezoid family is a strictly decreasing function of the smallest side-length $r_{34}=x$. The supremum of $\theta_{12}$ is $120^{\circ}$, from the degenerate equilateral triangle $\left(m_{3}=m_{4}=0\right.$ and $\left.r_{34}=0\right)$, while the minimum is $90^{\circ}$, attained at the square configuration. Similarly, the largest interior angle of the trapezoid is a strictly decreasing function of $x$, with a supremum of $120^{\circ}$ (degenerate equilateral triangle) and minimum of $90^{\circ}$ (square) while the smallest interior angle strictly increases from $60^{\circ}$ to $90^{\circ}$.

\section{Remarks:}

1. These bounds are the same as those for the co-circular kite family studied in Section 3.1.

2. In [15], a theorem stating that a unique isosceles trapezoid c.c. exists for each interior angle between $60^{\circ}$ and $120^{\circ}$ is presented, although no formal proof is given.

\section{The Surface of Co-Circular Central Configurations}

We now analyze the full set of co-circular central configurations and show it is equivalent to a graph over two of the exterior side-lengths. The set of interest is all vectors $\mathbf{r}=\left(1, r_{13}, r_{14}, r_{23}, r_{24}, r_{34}\right) \in \Omega$ satisfying equation (9), $P=0$ and $V=0$. Recall that for a cyclic quadrilateral, the diagonals can be expressed in terms of the exterior sides. Expanding the expressions in equation (15) yields

$$
\begin{aligned}
& r_{13}=\left(\frac{r_{14}^{2} r_{23}+r_{14} r_{34}\left(r_{23}^{2}+1\right)+r_{23} r_{34}^{2}}{r_{23}+r_{14} r_{34}}\right)^{1 / 2}, \\
& r_{24}=\left(\frac{r_{14}^{2} r_{23} r_{34}+r_{14}\left(r_{34}^{2}+r_{23}^{2}\right)+r_{23} r_{34}}{r_{14}+r_{23} r_{34}}\right)^{1 / 2},
\end{aligned}
$$

which are equivalent to $F_{1}=0$ and $F_{2}=0$, respectively. If equations (24) and (25) hold, one can check that both $P=0$ and $V=0$ follow. This avoids the need to use the Cayley-Menger determinant $V=0$ and also serves to eliminate the variables $r_{13}$ and $r_{24}$. Substituting relations (24) and (25) into equation (9) and setting $r_{12}=1$ yields

$$
F\left(r_{14}, r_{23}, r_{34}\right)=\left(r_{13}^{3}-1\right)\left(r_{23}^{3}-r_{34}^{3}\right)\left(r_{24}^{3}-r_{14}^{3}\right)-\left(1-r_{14}^{3}\right)\left(r_{24}^{3}-r_{34}^{3}\right)\left(r_{13}^{3}-r_{23}^{3}\right)=0
$$

where $r_{13}$ and $r_{24}$ are understood to be functions of $r_{14}, r_{23}, r_{34}$ given by equations (24) and (25), respectively.

The relations on the mutual distances in $\Omega$, coupled with the fact that the bodies are on a common circle, lead to further restrictions on the variables $r_{14}, r_{23}, r_{34}$. Since $r_{13}>1$, equation (24) implies that

$$
r_{14}^{2} r_{23}+r_{14} r_{34}\left(r_{23}^{2}+1\right)+r_{23} r_{34}^{2}>r_{23}+r_{14} r_{34}
$$



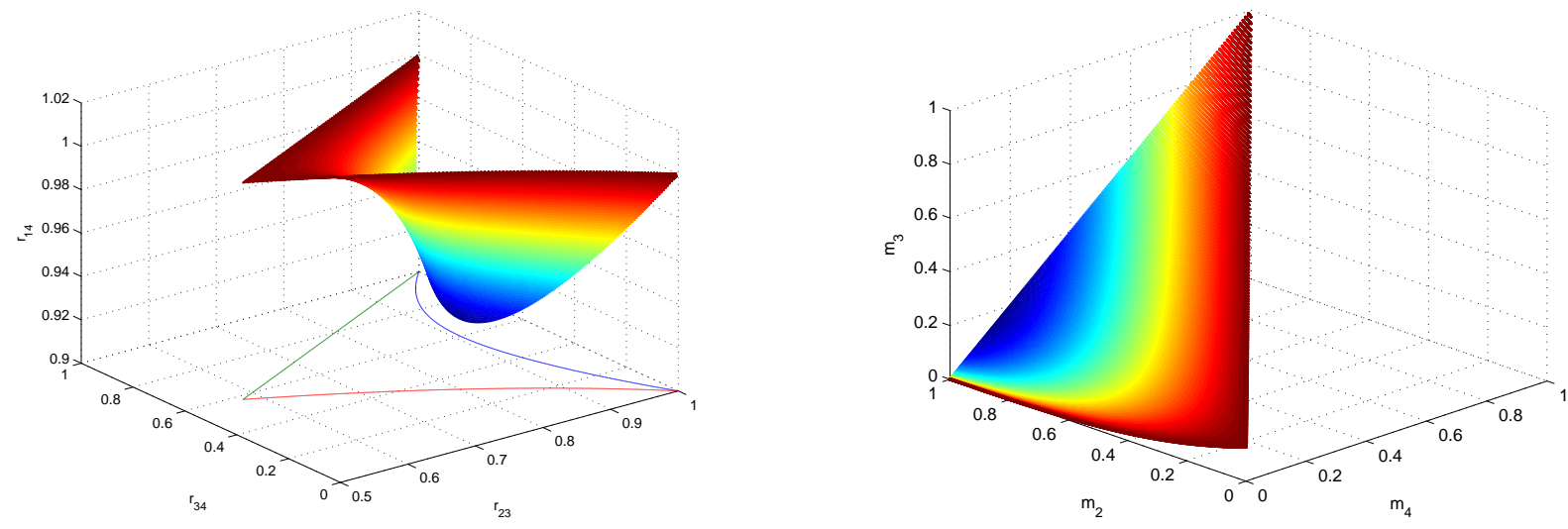

Figure 6: On the left is the surface $\Gamma$ of co-circular central configurations in $r_{34} r_{23} r_{14}$-space. The outline of the projection onto the $r_{34} r_{23}$-plane is shown plotted in the plane $r_{14}=0.9$. This figure was generated with Matlab [17] using a bisection algorithm. On the right is the image of $\Gamma$ in $m_{2} m_{3} m_{4}$-space under equations (16), (17) and (18) with $m_{1}=1$. This figure shows the full set of masses for which a co-circular c.c. exists.

which simplifies to

$$
r_{14}^{2}+r_{34}^{2}+r_{14} r_{34} r_{23}>1
$$

Similarly, $r_{24}>1$ and equation (25) implies that

$$
r_{23}^{2}+r_{34}^{2}+r_{14} r_{34} r_{23}>1 \text {. }
$$

Note that since we are assuming $r_{14} \geq r_{23}$, inequality (26) follows directly from inequality (27). Also, the calculations above can be reversed, so that inequalities (26) and (27) imply $r_{13}>1$ and $r_{24}>1$, respectively. We have proven the following lemma.

Lemma 4.1 Let

$$
\begin{aligned}
& \mathcal{C}=\left\{\left(r_{14}, r_{23}, r_{34}\right) \in \mathbb{R}^{+^{3}}: 1 \geq r_{14} \geq r_{23} \geq r_{34} \text { and } r_{23}^{2}+r_{34}^{2}+r_{14} r_{34} r_{23}>1\right\}, \text { and } \\
& \Gamma=\left\{\mathbf{s}=\left(r_{14}, r_{23}, r_{34}\right) \in \mathbb{R}^{+^{3}}: \mathbf{s} \in \mathcal{C} \text { and } F(\mathbf{s})=0\right\} .
\end{aligned}
$$

Any point in $\Gamma$ corresponds to a four-body co-circular central configuration with positive masses and up to a relabeling and rescaling, $\Gamma$ contains all such configurations.

\section{1 $\Gamma$ as a Graph}

We now show that $\Gamma$ is a surface by proving that it is a graph $r_{14}=f\left(r_{23}, r_{34}\right)$ (see Figure 6). First note that the boundaries of $\Gamma$ consist of a line corresponding to the kites (K), a curve containing the isosceles trapezoids $(\mathrm{T})$ and a curve corresponding to degenerate central configurations (D) 
with $m_{3}=0$. Since they contain only positive masses, $(\mathrm{K})$ and $(\mathrm{T})$ are included in $\Gamma$ while (D) is excluded. The kites lie on the line $r_{14}=1, r_{23}=r_{34}=x$ with $1 / \sqrt{3}<x \leq 1$. The trapezoids lie on a curve in the plane $r_{14}=r_{23}$ and as shown in Section 3.2, $r_{23}$ can be written as a differentiable function of $r_{34}$. The degenerate central configurations with $m_{3}=0$ are critical points of the planar, circular, restricted four-body problem. Here, bodies 1, 2 and 4 lie on an equilateral triangle, with $r_{12}=r_{24}=r_{14}=1$. It is straight-forward to show that if $r_{24}=1$, that is, when the only strict inequality defining $\mathcal{C}$ becomes an equality, then $F=0$ can only be satisfied on $\mathcal{C}$ if $r_{14}=1$. Therefore, the boundary of $\Gamma$ contains only the cases $(K),(T)$ or $(D)$.

Lemma 4.2 On $\mathcal{C}$, both $\frac{\partial r_{13}}{\partial r_{14}}$ and $\frac{\partial r_{24}}{\partial r_{14}}$ are strictly positive.

Proof: A straight-forward calculation shows that

$$
\frac{\partial r_{13}}{\partial r_{14}}=\frac{r_{23}\left[r_{34}\left(r_{14}^{2}+r_{23}^{2}-r_{34}^{2}+1\right)+2 r_{14} r_{23}\right]}{2 r_{13}\left(r_{23}+r_{14} r_{34}\right)^{2}}
$$

which is strictly positive on $\mathcal{C}$ since $r_{23} \geq r_{34}$. A similar calculation yields

$$
\frac{\partial r_{24}}{\partial r_{14}}=\frac{r_{23} r_{34}\left(r_{14}^{2}+r_{23}^{2}+r_{34}^{2}+2 r_{14} r_{23} r_{34}-1\right)}{2 r_{24}\left(r_{14}+r_{23} r_{34}\right)^{2}}
$$

which is strictly positive due to inequality (27).

Recall that for the isosceles trapezoid family, the exterior side $r_{23}$ can be written as a differentiable function of $r_{34}$, denoted by $r_{23}=\tau\left(r_{34}\right)$ for $0 \leq r_{34} \leq 1$ and that $\tau<1$ except for $r_{34}=0,1$.

Theorem 4.3 The set of co-circular central configurations $\Gamma$ is the graph of a differentiable function $r_{14}=f\left(r_{34}, r_{23}\right)$ over the two variables $r_{34}$ and $r_{23}$. The domain of this function is the region

$$
\mathcal{D}=\left\{\left(r_{34}, r_{23}\right) \in \mathbb{R}^{+^{2}}: 1 \geq r_{23} \geq r_{34}, r_{23} \leq \tau\left(r_{34}\right) \text { and } r_{23}^{2}+r_{34}^{2}+r_{34} r_{23}>1\right\} .
$$

Proof: We will show that the projection of $\Gamma$ onto the $r_{34} r_{23}$-plane equals $\mathcal{D}$ (see Figure 7 ) and that $r_{14}$ can be written as a differentiable function of $r_{34}$ and $r_{23}$ over $\mathcal{D}$, that is, $\frac{\partial f}{\partial r_{34}}$ and $\frac{\partial f}{\partial r_{23}}$ exist and are continuous on the interior of $\mathcal{D}$.

According to the definition of $\mathcal{C}$, the distance $r_{14}$ must satisfy both

$$
r_{14} \geq r_{23} \quad \text { and } \quad r_{14}>\frac{1-r_{23}^{2}-r_{34}^{2}}{r_{23} r_{34}}
$$

Therefore, define

$$
\hat{z}\left(r_{34}, r_{23}\right)=\max \left\{r_{23}, \frac{1-r_{23}^{2}-r_{34}^{2}}{r_{23} r_{34}}\right\} .
$$

We will show that for a given point $\left(r_{34}, r_{23}\right)$ in $\mathcal{D}, F=0$ has a unique solution for some $r_{14}$ satisfying $\hat{z} \leq r_{14} \leq 1$. This point will clearly lie in $\Gamma$. 


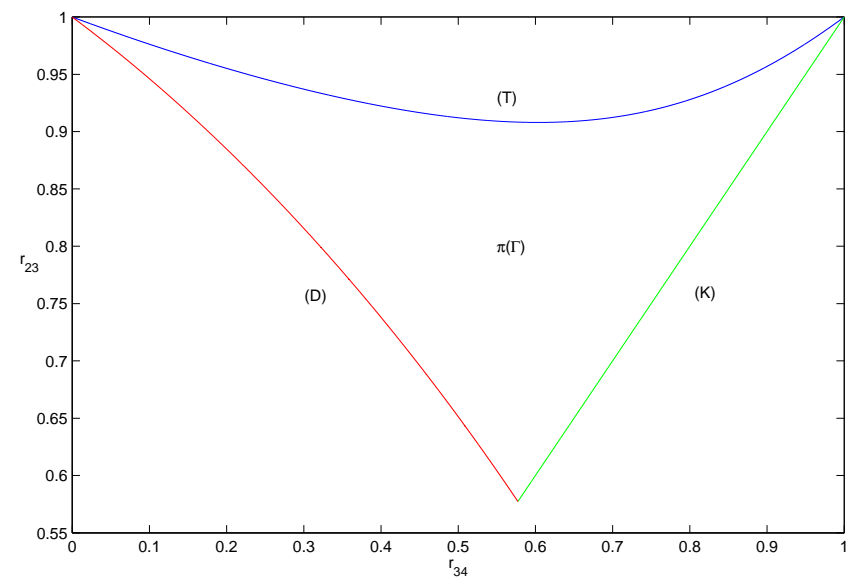

Figure 7: The projection $\pi(\Gamma)=\mathcal{D}$ of the surface of co-circular c.c.'s onto the $r_{34} r_{23}$-plane.

The intersection of the two surfaces $r_{14}=r_{23}$ and $r_{14}=\left(1-r_{23}^{2}-r_{34}^{2}\right) /\left(r_{23} r_{34}\right)$ projected onto the first quadrant of the $r_{34} r_{23}$-plane is a piece of the parabola $r_{23}^{2}=1-r_{34}$. This curve divides $\mathcal{D}$ into two regions, denoted $D_{1}$ and $D_{2}$. On $D_{1}$, defined as the set of points in the interior of $\mathcal{D}$ for which $r_{23}<\sqrt{1-r_{34}}$, we have that $\hat{z}=\left(1-r_{23}^{2}-r_{34}^{2}\right) /\left(r_{23} r_{34}\right)$. On $D_{2}$, defined as the set of points in the interior of $\mathcal{D}$ for which $r_{23} \geq \sqrt{1-r_{34}}$, we have that $\hat{z}=r_{23}$.

The three curves defining the boundaries of $\mathcal{D}$ correspond to the kites $\left(r_{23}=r_{34}\right)$, the isosceles trapezoid family $\left(r_{23}=\tau\left(r_{34}\right)\right)$ and the degenerate case with $m_{3}=0\left(r_{24}=r_{14}=1\right)$. If $r_{14}=1$ and we restrict to the interior of $\mathcal{D}$, then inequalities (26) and (27) show that $r_{13}>1$ and $r_{24}>1$. It then follows that

$$
F\left(r_{14}=1, r_{23}, r_{34}\right)=\left(r_{13}^{3}-1\right)\left(r_{24}^{3}-1\right)\left(r_{23}^{3}-r_{34}^{3}\right),
$$

is strictly positive on the interior of $\mathcal{D}$.

Next, we claim that $F\left(r_{14}=\hat{z}, r_{23}, r_{34}\right)$ is strictly negative on the interior of $\mathcal{D}$. To see this, consider a point in the region $D_{1}$ and suppose that $r_{14}=\hat{z}=\left(1-r_{23}^{2}-r_{34}^{2}\right) /\left(r_{23} r_{34}\right)$. Then, inequality (27) becomes an equality and $r_{24}=1$. Moreover, $0<r_{23} \leq r_{14}<1$ by the definition of $\hat{z}$ and the fact that $\left(r_{34}, r_{23}\right)$ is in the interior of $\mathcal{D}$. We also have, using equation $(24)$, that

$$
r_{13}^{2}-r_{34}^{2}=\frac{r_{14}\left(r_{14} r_{23}+r_{34}+r_{34}\left(r_{23}^{2}-r_{34}^{2}\right)\right)}{r_{23}+r_{14} r_{34}}>0
$$

since $r_{23}>r_{34}$. It follows that

$$
F\left(r_{14}=\hat{z}, r_{23}, r_{34}\right)=-\left(1-r_{14}^{3}\right)\left(1-r_{23}^{3}\right)\left(r_{13}^{3}-r_{34}^{3}\right)
$$

is strictly negative on $D_{1}$.

Now consider a point in $D_{2}$ and suppose that $r_{14}=r_{23}$. On this plane, we also have that $r_{13}=r_{24}$ since the quadrilateral is cyclic (or by examining equations (24) and (25)). A short computation gives

$$
F\left(r_{14}=r_{23}, r_{23}, r_{34}\right)=\left(r_{13}^{3}-r_{23}^{3}\right) \cdot T\left(x=r_{34}, y=r_{23}\right)
$$


where $T$ is given by equation (21) from the isosceles trapezoid family. As demonstrated in the proof of Lemma 3.3, the value of $T$ on $D_{2}$ is strictly negative since $T$ vanishes only on the upper boundary of $\mathcal{D}$ and $\frac{\partial T}{\partial y}(x, y=\tau(x))>0$. Since $r_{14}=r_{23}$ implies that $r_{13}=\sqrt{r_{34}+r_{23}^{2}}>r_{23}$, we have that $F\left(r_{14}=r_{23}, r_{23}, r_{34}\right)$ is strictly negative. This shows that $F\left(r_{14}=\hat{z}, r_{23}, r_{34}\right)$ is strictly negative on the interior of $\mathcal{D}$. By the intermediate value theorem, it follows that for each point $\left(r_{34}, r_{23}\right)$ in the interior of $\mathcal{D}$, there exists a solution to $F=0$ for some $r_{14}$ satisfying $\hat{z}<r_{14}<1$.

To see that this solution is unique, we show that $\frac{\partial F}{\partial r_{14}}>0$ on $\Gamma$. We compute that

$$
\frac{\partial F}{\partial r_{14}}=3 r_{13}^{2} \frac{\partial r_{13}}{\partial r_{14}} \alpha_{1}+3 r_{24}^{2} \frac{\partial r_{24}}{\partial r_{14}} \alpha_{2}+3 r_{14}^{2} \alpha_{3}
$$

where

$$
\begin{aligned}
& \alpha_{1}=\left(r_{23}^{3}-r_{34}^{3}\right)\left(r_{24}^{3}-r_{14}^{3}\right)-\left(1-r_{14}^{3}\right)\left(r_{24}^{3}-r_{34}^{3}\right), \\
& \alpha_{2}=\left(r_{23}^{3}-r_{34}^{3}\right)\left(r_{13}^{3}-1\right)-\left(1-r_{14}^{3}\right)\left(r_{13}^{3}-r_{23}^{3}\right), \\
& \alpha_{3}=\left(r_{24}^{3}-r_{34}^{3}\right)\left(r_{13}^{3}-r_{23}^{3}\right)-\left(r_{23}^{3}-r_{34}^{3}\right)\left(r_{13}^{3}-1\right) .
\end{aligned}
$$

Using $F=0$, each $\alpha_{i}$ restricted to $\Gamma$ simplifies to

$$
\begin{aligned}
\alpha_{1} & =\frac{\left(1-r_{14}^{3}\right)\left(r_{24}^{3}-r_{34}^{3}\right)\left(1-r_{23}^{3}\right)}{r_{13}^{3}-1} \\
\alpha_{2} & =\frac{\left(1-r_{14}^{3}\right)\left(r_{13}^{3}-r_{23}^{3}\right)\left(r_{14}^{3}-r_{34}^{3}\right)}{r_{24}^{3}-r_{14}^{3}} \\
\alpha_{3} & =\frac{\left(r_{24}^{3}-r_{34}^{3}\right)\left(r_{13}^{3}-r_{23}^{3}\right)\left(r_{24}^{3}-1\right)}{r_{24}^{3}-r_{14}^{3}}
\end{aligned}
$$

It is clear that $\alpha_{3}$ is strictly positive on $\Gamma$ while $\alpha_{1}$ and $\alpha_{2}$ are non-negative. Taken together with Lemma 4.2 , this proves that $\frac{\partial F}{\partial r_{14}}>0$ on $\Gamma$. Hence, there exists a function $r_{14}=f\left(r_{34}, r_{23}\right)$ implicitly defined by $F=0$ over the interior of $\mathcal{D}$.

Finally, on the boundary of $\mathcal{D}$ defined by $r_{23}=r_{34}$, the only possible solution to $F=0$ that lies inside $\mathcal{C}$ is $r_{14}=1$ (the kite configurations). Similarly, on the boundary $r_{23}=\tau\left(r_{34}\right)$, since $\frac{\partial F}{\partial r_{14}}>0$, the only possible solution to $F=0$ that lies inside $\mathcal{C}$ is $r_{14}=r_{23}$ (the trapezoid family). Thus, we define $f\left(r_{34}, r_{23}=r_{34}\right)=1$ and $f\left(r_{34}, r_{23}=\tau\left(r_{34}\right)\right)=r_{23}$ to extend $f$ to all of $\mathcal{D}$. The implicit function theorem, which is applicable on the boundaries (K) and (T) as well, and the fact that $F=0$ is algebraic, then shows that $f$ is continuous on all of $\mathcal{D}$ and differentiable on the interior of $\mathcal{D}$.

From the proof of Lemma 3.3, any point $\left(r_{34}, r_{23}\right)$ lying above the curve $\tau$ has $T>0$. Since $\frac{\partial F}{\partial r_{14}}>0$ on $\Gamma$, it follows that the solution to $F=0$ must satisfy $r_{14}<r_{23}$, which lies outside $\mathcal{C}$. Likewise, any point $\left(r_{34}, r_{23}\right)$ lying on or below the curve $r_{23}^{2}+r_{34}^{2}+r_{34} r_{23}=1$, will have $r_{24} \leq 1$, which also lies outside $\mathcal{C}$. It follows that the projection of $\Gamma$ onto the $r_{34} r_{23}$-plane is precisely equal to $\mathcal{D}$. 


\subsection{Ordering of the Masses}

Remarkably, it is not difficult to show that for any co-circular central configuration, the masses must be ordered in a precise fashion. The arguments that follow rely mostly on the fact that the four bodies lie on a common circle, as well as our ordering of the mutual distances.

Theorem 4.4 Any co-circular central configuration in $\Gamma$ satisfies

$$
m_{3} \leq m_{4} \leq m_{2} \leq m_{1}=1
$$

In other words, the largest body is located at the vertex between the two longest exterior sides, and the smallest body is opposite the largest one. In addition, the two largest bodies lie on the longest side while the two smallest bodies lie on the smallest side.

Before proving this theorem, we verify some important inequalities relating the two diagonals with the second and third-longest exterior sides.

Lemma 4.5 Any co-circular central configuration in $\Gamma$ satisfies

$$
\begin{aligned}
& r_{13}-r_{24} \leq\left(r_{14}-r_{23}\right)\left(1-r_{34}\right), \quad \text { and } \\
& r_{13}-r_{24} \leq r_{14}-r_{23} .
\end{aligned}
$$

Proof: Using equation (15), we have

$$
r_{13}-r_{24}=\sqrt{\frac{a}{b c}}(b-c)=\sqrt{\frac{a}{b c}}\left(r_{14}-r_{23}\right)\left(1-r_{34}\right) .
$$

Then, since

$$
\begin{aligned}
b c & =\left(r_{14}+r_{23} r_{34}\right)\left(r_{23}+r_{14} r_{34}\right) \\
& =r_{14} r_{23}+r_{34}\left(r_{14}^{2}+r_{23}^{2}+r_{14} r_{23} r_{34}\right) \\
& \left.>r_{14} r_{23}+r_{34} \quad \text { (by the definition of } \mathcal{C}\right) \\
& =a,
\end{aligned}
$$

we have $\frac{a}{b c}<1$, which verifies inequality (29). Since $0<r_{34} \leq 1$, inequality (30) immediately follows from (29). We note that equality is also needed in both (29) and (30) due to the isosceles trapezoid family where both sides vanish. In fact, these inequalities are strict for all other co-circular c.c.'s.

Lemma 4.6 Any co-circular central configuration in $\Gamma$ satisfies

$$
\frac{r_{13}}{r_{24}} \leq \frac{r_{14}}{r_{23}}
$$


Proof: Since $r_{23} \leq r_{14}$, we have

$$
\frac{r_{13}}{r_{24}}=\frac{r_{14}+r_{23} r_{34}}{r_{23}+r_{14} r_{34}} \leq \frac{r_{14}\left(1+r_{34}\right)}{r_{23}\left(1+r_{34}\right)}=\frac{r_{14}}{r_{23}}
$$

Proof of Theorem 4.4: We begin by showing

$$
m_{2}=\frac{r_{23}^{2} r_{24}^{2}\left(r_{13}^{3}-r_{14}^{3}\right)}{r_{13}^{2} r_{14}^{2}\left(r_{24}^{3}-r_{23}^{3}\right)} \leq 1
$$

Since $r_{13} \geq r_{24}$ and $r_{14} \geq r_{23}$, it suffices to show that $r_{24}^{3}-r_{23}^{3} \geq r_{13}^{3}-r_{14}^{3}$ or equivalently,

$$
r_{13}^{3}-r_{24}^{3} \leq r_{14}^{3}-r_{23}^{3} .
$$

Cubing both sides of inequality (30) yields

$$
r_{13}^{3}-r_{24}^{3}+\left(-3 r_{13}^{2} r_{24}+3 r_{13} r_{24}^{2}+3 r_{14}^{2} r_{23}-3 r_{14} r_{23}^{2}\right) \leq r_{14}^{3}-r_{23}^{3} .
$$

Inequality (31) will then follow, provided $-r_{13}^{2} r_{24}+r_{13} r_{24}^{2}+r_{14}^{2} r_{23}-r_{14} r_{23}^{2}$ is non-negative. Factoring, this is equivalent to showing

$$
r_{13} r_{24}\left(r_{24}-r_{13}\right)+r_{14} r_{23}\left(r_{14}-r_{23}\right) \geq 0 .
$$

Beginning with $1 \leq r_{13} r_{24}=r_{23} r_{14}+r_{34}$, we have the following sequence of implications:

$$
\begin{aligned}
1 \leq r_{34}+r_{14} r_{23} & \Longrightarrow r_{34}-r_{34}^{2}-r_{34} r_{14} r_{23} \leq 0 \\
& \Longrightarrow\left(1-r_{34}\right)\left(r_{14} r_{23}+r_{34}\right) \leq r_{14} r_{23} \\
& \Longrightarrow r_{13} r_{24}\left(1-r_{34}\right) \leq r_{14} r_{23} \\
& \Longrightarrow r_{13} r_{24}\left(r_{14}-r_{23}\right)\left(1-r_{34}\right) \leq r_{14} r_{23}\left(r_{14}-r_{23}\right) .
\end{aligned}
$$

Then, using inequality (29), we have

$$
r_{13} r_{24}\left(r_{13}-r_{24}\right) \leq r_{13} r_{24}\left(r_{14}-r_{23}\right)\left(1-r_{34}\right) \leq r_{14} r_{23}\left(r_{14}-r_{23}\right)
$$

which verifies inequality (32) and shows that $m_{2} \leq 1$.

Next, we verify that $m_{4} \leq m_{2}$. Using equations (16) and (18), we have

$$
\frac{m_{2}}{m_{4}}=\frac{r_{23}^{2}}{r_{14}^{2} r_{34}^{2}} \cdot \frac{r_{13}^{3}-r_{14}^{3}}{r_{13}^{3}-1} \cdot \frac{r_{24}^{3}-r_{34}^{3}}{r_{24}^{3}-r_{23}^{3}}
$$

Each of the three fractions in equation (33) are greater than or equal to 1 . This follows since $r_{23} \geq r_{34}$ and $1 \geq r_{14}$. Consequently, $m_{2} / m_{4} \geq 1$, as desired.

Finally, using equations (17), (18) and (9), we compute

$$
\frac{m_{4}}{m_{3}}=\frac{r_{24}^{2} r_{14}^{2}\left(r_{13}^{3}-1\right)\left(r_{23}^{3}-r_{34}^{3}\right)}{r_{13}^{2} r_{23}^{2}\left(r_{24}^{3}-r_{34}^{3}\right)\left(1-r_{14}^{3}\right)}=\frac{r_{24}^{2} r_{14}^{2}\left(r_{13}^{3}-r_{23}^{3}\right)}{r_{13}^{2} r_{23}^{2}\left(r_{24}^{3}-r_{14}^{3}\right)} \text {. }
$$


By Lemma 4.6, $r_{24} r_{14} \geq r_{13} r_{23}$. Since $r_{13} \geq r_{24}$ and $r_{14} \geq r_{23}$, we also have $r_{13}^{3}-r_{23}^{3} \geq r_{24}^{3}-r_{14}^{3}$. This proves that $m_{3} \leq m_{4}$.

Somewhat surprisingly, Theorem 4.4 and its proof give strong implications if just two of the masses are equal.

Corollary 4.7 If just two bodies of a co-circular central configuration have equal mass, then the configuration is symmetric, either a kite or an isosceles trapezoid. Specifically, for any co-circular c.c. in $\Gamma$, if either $m_{1}=m_{2}$ or $m_{3}=m_{4}$, then the configuration is an isosceles trapezoid and the other pair of masses are necessarily equal. If $m_{2}=m_{4}$, the configuration is a kite. If any three masses are equal, then the configuration is a square and all four masses are necessarily equal.

\section{Proof:}

From the proof for $m_{2} \leq 1$, we have that

$$
m_{2}=\frac{r_{23}^{2}}{r_{14}^{2}} \cdot \frac{r_{24}^{2}}{r_{13}^{2}} \cdot \frac{r_{13}^{3}-r_{14}^{3}}{r_{24}^{3}-r_{23}^{3}}
$$

is the product of three positive numbers less than or equal to one. If $m_{2}=m_{1}=1$, it follows that each of the fractions in equation (34) is equal to one. This quickly yields $r_{14}=r_{23}$ and $r_{13}=r_{24}$, yielding an isosceles trapezoid. Then, by Theorem 3.2, $m_{3}=m_{4}$ follows.

Next, suppose that $m_{3}=m_{4}$. Then, from the proof for $m_{3} \leq m_{4}$, we must have $r_{13}^{3}-r_{23}^{3}=$ $r_{24}^{3}-r_{14}^{3}$. This implies that

$$
r_{13}^{3}-r_{24}^{3}=r_{23}^{3}-r_{14}^{3} .
$$

If $r_{23}<r_{14}$, then the right-hand side of equation (35) is negative, contradicting the fact that $r_{13} \geq r_{24}$. Thus, $r_{23}=r_{14}$ and then equation (35) gives $r_{13}=r_{24}$ and the configuration is, once again, an isosceles trapezoid. Then, by Theorem 3.2, $m_{1}=m_{2}$ follows.

Finally, if $m_{2}=m_{4}$, a similar argument using equation (33) shows that $r_{14}=1$ and $r_{23}=r_{34}$, yielding a kite configuration. If three of the masses are equal (either $m_{3}=m_{4}=m_{2}$ or $m_{4}=m_{2}=$ $m_{1}$ ), then the co-circular c.c. is simultaneously both an isosceles trapezoid and a kite. This means it is a square and that all four masses must be equal.

\section{Remarks:}

1. Corollary 4.7 shows that if one pair of masses on either the longest or shortest exterior side are equal, then the other pair is also equal and the configuration must be an isosceles trapezoid. One would expect that with the same assumption, such a strong conclusion does not hold for generic four-body convex central configurations.

2. The fact that $m_{2}=m_{4}$ implies the configuration is a kite is already a consequence of the main theorem in [4], where it is shown that this fact is true for any convex c.c., not just co-circular ones. 

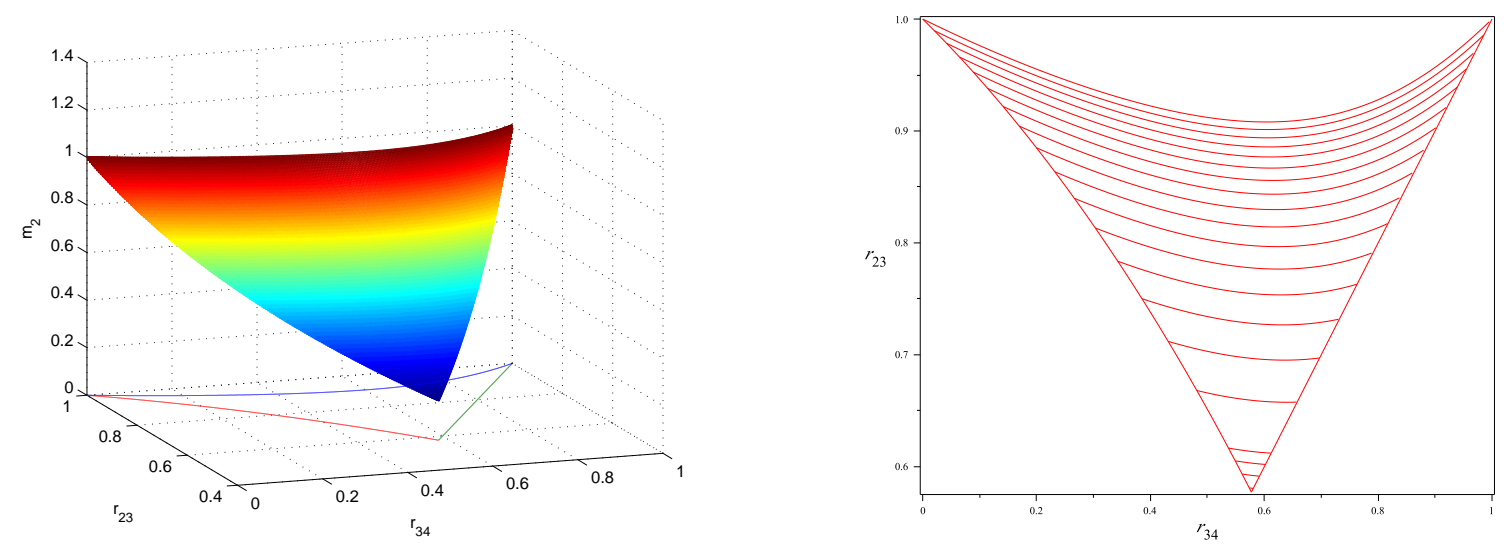

Figure 8: Surface and contour plots of the mass $m_{2}$ as a function of the exterior side-lengths $r_{23}$ and $r_{34}$.

3. Corollary 4.7 and our work on the symmetric cases shows that there are positive masses for which no co-circular central configuration exists. For example, if we consider a set of masses where $m_{i}=m_{j}$ for some choice of indices, then the configuration must be symmetric. However, our work in Section 3 shows that there is a specific relationship between the other two masses to insure that the configuration is a c.c.c. Thus, generically choosing the remaining two masses will not yield a co-circular central configuration, regardless of relabeling or rescaling.

4. In the four-body problem, for a given choice and ordering of the masses, there exists a convex central configuration $[15,30]$. It is not known whether this configuration is unique. If we restrict to co-circular c.c.'s, we conjecture that the configuration is indeed unique. However, a global argument on all of $\Gamma$ seems technically quite challenging. We present some visual evidence for uniqueness in Figures 8 and 9. By contradiction, if there existed a choice of masses for which two distinct c.c.c.'s existed, then one of the curves in Figure 9 would have to intersect itself or collapse to a point. This does not appear to be the case.

5. The right-hand graph in Figure 6 shows the surface of masses in $m_{2} m_{3} m_{4}$-space for which a co-circular c.c. exists. Attempts using Gröbner bases and symbolic computation software to find a single polynomial expression relating the three masses were unsuccessful. However, if $I=<F_{1}, F_{2}, F_{3}>\subset \mathbb{C}\left[r_{13}, r_{14}, r_{23}, r_{24}, r_{34}\right]$ is the ideal generated by the first three equations in System I, then it is possible (using Maple) to compute a Gröbner basis for $I$ using a graded reverse lexicographic order. From this calculation, we deduce that the dimension of the algebraic variety of $I$ is two. Then, since the equations for $m_{2}, m_{3}$ and $m_{4}$ are rational functions of the distance variables, it follows that the image of $\Gamma$ under the three mass functions is contained in a two-dimensional algebraic variety in $\mathbb{R}\left[m_{2}, m_{3}, m_{4}\right]$. Consequently, for a generic choice of positive masses, there does not exist a co-circular central configuration. We thank John Little for providing the details for this argument. 

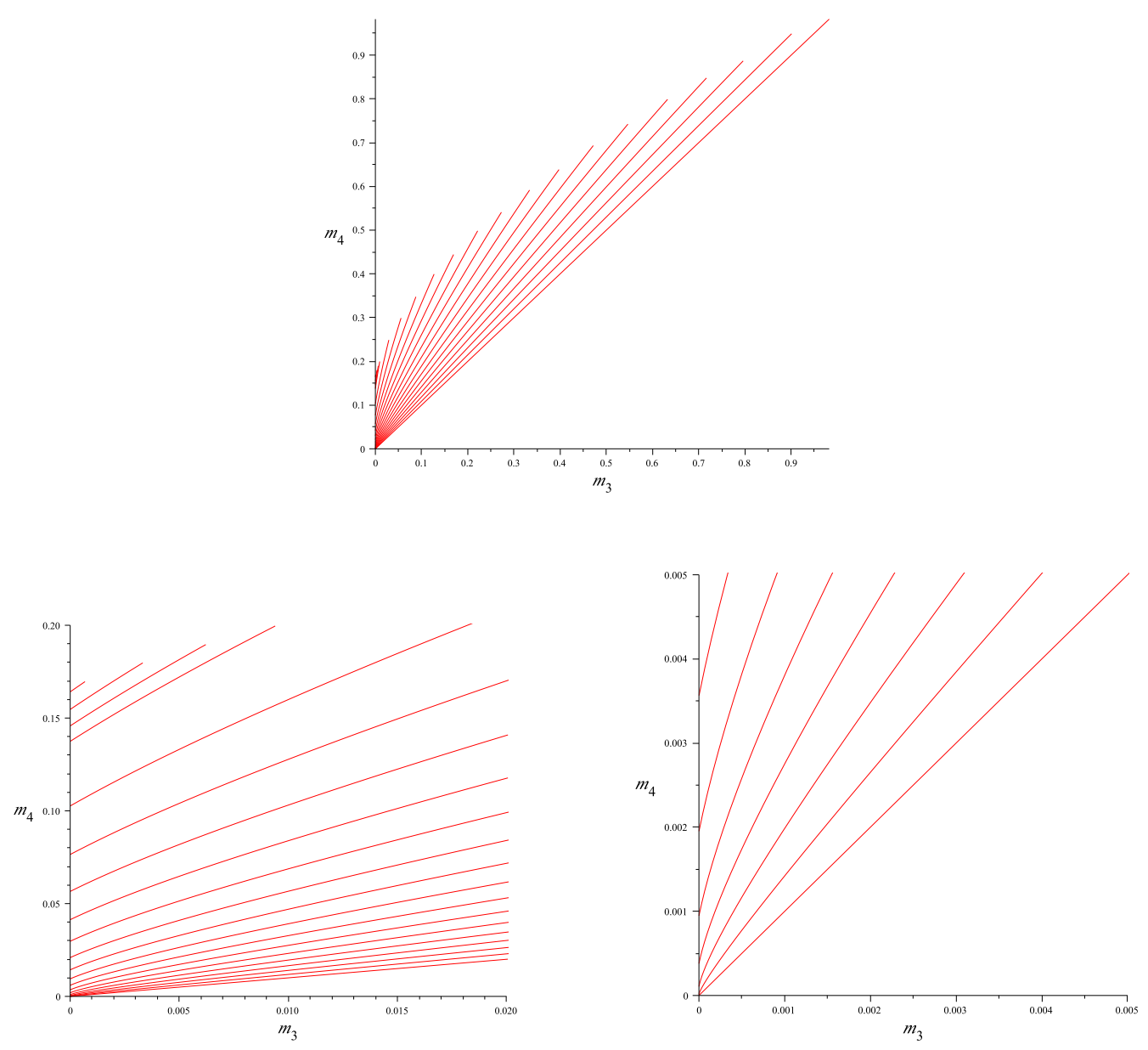

Figure 9: Each curve shows the relationship between $m_{3}$ and $m_{4}$ along a particular level curve of $m_{2}=$ constant. The bottom two figures are magnifications near the origin of the top figure.

\section{Some Geometric Facts about Co-Circular C.C.'s}

We now provide some precise bounds on the mutual distances as well as some interesting geometric facts about four-body co-circular central configurations.

\subsection{Bounds on the Mutual Distances}

Let $\hat{\tau}$ be the minimum value of the function $\tau(x)$ defined in Section 3.2 for the isosceles trapezoid family. From Remark 3 after Lemma $3.3, \hat{\tau} \approx 0.908026$. It turns out that this value is also the minimum value for $r_{14}$ on all of $\Gamma$.

Proposition 5.1 The exterior sides and the diagonals of a co-circular central configuration in $\Gamma$ 
are restricted by

$$
0<r_{34} \leq 1, \quad \frac{1}{\sqrt{3}}<r_{23} \leq 1, \quad \hat{\tau} \leq r_{14} \leq 1, \quad 1<r_{13}, r_{24} \leq \sqrt{2}
$$

These bounds are sharp.

Proof: The bounds for the distances $r_{34}$ and $r_{23}$ follow directly from Theorem 4.3. Using a similar argument as in the proof of Theorem 4.3, we have that $\frac{\partial F}{\partial r_{23}}>0$ on the interior of $\mathcal{D}$. By the implicit function theorem, it follows that $\frac{\partial r_{14}}{\partial r_{23}}<0$ on the interior of $\mathcal{D}$. Since $r_{14}=1$ on the lower boundaries of $\mathcal{D}$ (the degenerate equilateral triangle or kite families), it follows that the minimum value for $r_{14}$ must occur on the upper boundary of $\mathcal{D}$ given by the curve $r_{23}=\tau\left(r_{34}\right)$. However, on this curve we have $r_{14}=r_{23}$ (the isosceles trapezoid case). Therefore, the minimum value of $r_{14}$ occurs at the minimum value of $r_{23}$ on $\tau$. This is precisely the number $\hat{\tau} \approx 0.908026$.

The lower bounds on the diagonals come directly from the definition of $\Gamma$ and follow from inequality (27). To see that they are sharp, note that as $\left(r_{34}, r_{23}\right)$ approaches the lower-left boundary curve of $\mathcal{D}, r_{34}^{2}+r_{23}^{2}+r_{34} r_{23}=1, r_{14}$ approaches one due to the second inequality in (28). Then, inequality (27) becomes an equality and $r_{24}$ approaches one. The diagonal $r_{13}$ can also become arbitrarily close to one in the upper-left corner of $\mathcal{D}$. Here, we have $r_{34}$ approaching zero and both $r_{23}$ and $r_{14}$ approaching one. Equation (24) then shows that $r_{13}$ also approaches one.

Next, by equation (15), $r_{13} \leq \sqrt{2}$ is equivalent to $a b \leq 2 c$ or

$$
\left(r_{34}+r_{14} r_{23}\right)\left(r_{14}+r_{23} r_{34}\right) \leq 2\left(r_{23}+r_{14} r_{34}\right) .
$$

This, in turn, can be written as

$$
r_{14} r_{34}\left(1-r_{23}^{2}\right) \geq r_{23}\left(r_{14}^{2}+r_{34}^{2}-2\right) .
$$

Since $r_{14} \leq 1$ and $r_{34} \leq 1$, the right-hand side of (36) is non-positive, while $r_{23} \leq 1$ implies that the left-hand side of (36) is non-negative. This verifies (36) and proves that $r_{13} \leq \sqrt{2}$. Since $r_{24} \leq r_{13}$, the upper-bound on $r_{24}$ is also established. These bounds are attained, but only at the square configuration, since $r_{23}=r_{34}=r_{14}=1$ is the only possible way to make inequality (36) an equality.

For a general four-body convex central configuration with diagonals $r_{13}$ and $r_{24}$, and $r_{13} \geq r_{24}$, a straight-forward geometric argument shows that $1 \leq \frac{r_{13}}{r_{24}}<\sqrt{3}$. This is part of Theorem 4.1 in [24]. Requiring the bodies to lie on a common circle reduces this bound considerably. More precisely, the ratio $r_{13} / r_{24}$ of the diagonals satisfies the following sharp bounds:

$$
1 \leq \frac{r_{13}}{r_{24}}<\frac{2}{\sqrt{3}} \approx 1.1547
$$

This follows because $\partial\left(\frac{r_{13}}{r_{24}}\right) / \partial r_{23}<0$ on the interior of $\mathcal{D}$. The minimum value of the ratio of the diagonals (one) occurs at the upper boundary of $\mathcal{D}$, at the isosceles trapezoid case $(\mathrm{T})$, while the maximum value occurs at $r_{34}=r_{23}=1 / \sqrt{3}$, the point of intersection of the kite family $(\mathrm{K})$ and the family of degenerate central configurations (D). 
Furthermore, the ratio of the two opposite exterior sides $r_{14}$ and $r_{23}$ satisfies the following sharp bounds:

$$
1 \leq \frac{r_{14}}{r_{23}}<\sqrt{3}
$$

This follows since $\partial\left(\frac{r_{14}}{r_{23}}\right) / \partial r_{23}<0$ on the interior of $\mathcal{D}$. Once again, the minimum value of the ratio in question occurs at the upper boundary of $\mathcal{D}$. This value is one, since $r_{14}=r_{23}$ on the boundary (T). The maximum occurs at the lower boundary of $\mathcal{D}$ where $r_{14}=1$ for both (K) and (D). Thus, $\frac{r_{14}}{r_{23}}=\frac{1}{r_{23}}$ has a supremum of $\sqrt{3}$, since the infimum of $r_{23}$ is $1 / \sqrt{3}$.

It is interesting to note that while the largest values of $\frac{r_{13}}{r_{24}}$ and $\frac{r_{14}}{r_{23}}$ occur very close to the point of intersection between the kite and degenerate cases, the larger values of the ratio $\frac{r_{12}}{r_{34}}=\frac{1}{r_{34}}$ occur near the intersection of the trapezoid and degenerate cases (upper-left corner of $\mathcal{D}$ ). However, this ratio has no upper bound since $r_{34}$ can be arbitrarily small.

\subsection{Interior Angles, Arcs and Semi-circles}

According to Long [14], any interior angle of a four-body convex c.c. must lie between $60^{\circ}$ and $150^{\circ}$. Since opposite angles of a cyclic quadrilateral are supplementary, we can amend this bound for our problem. In fact, the maximum interior angle of a four-body co-circular central configuration lies between $90^{\circ}$ and $120^{\circ}$ while the minimum interior angle lies between $60^{\circ}$ and $90^{\circ}$. These bounds are sharp as they are identical to those obtained for the kite and trapezoid families.

A few restrictions on the possible shape and angles of a co-circular central configuration follow nicely from the perpendicular bisector theorem due to Conley. Suppose that $\mathbf{x}$ is a planar central configuration. For any pair of bodies $\mathbf{x}_{i}, \mathbf{x}_{j}$, consider the perpendicular bisector of the line segment joining the two bodies. Taken with the line passing through $\mathbf{x}_{i}$ and $\mathbf{x}_{j}$, a coordinate system is formed dividing the plane into four quadrants. The union of two opposite quadrants (taking the first with the third quadrant or the second with the fourth) excluding the axes forms an open cone. The perpendicular bisector theorem states that if one of these cones contains a body of the configuration, then so must the other cone. An elegant proof can be found in Moeckel's nice paper on central configurations [20].

As in Section 3, denote $\theta_{12}$ as the arc along the circumscribing circle between bodies 1 and 2 . By construction, this is the largest possible arc between consecutive bodies. Using the perpendicular bisector theorem and some simple geometry, it is possible to show that an upper bound for $\theta_{12}$ is $144^{\circ}$. However, numerical calculations suggest that this bound can be decreased to $120^{\circ}$ and that the value of $\theta_{12}$ decreases as $r_{23}$ increases through $\mathcal{D}$. The maximum $\theta_{12}$-value occurs at a minimum of the circumradius. Both the kite and trapezoid families have a supremum of $120^{\circ}$. Since $\theta_{12}$ is the largest arc along the circumscribing circle, it follows that the minimum value of $\theta_{12}$ is $90^{\circ}$, attained at the square configuration.

Moreover, using the perpendicular bisector theorem, it is straight-forward to see that no cocircular central configuration can lie entirely in a semi-circle. This result easily extends to any number of bodies, not just four-body co-circular central configurations. Finally, using the law of cosines and the definition of $\Gamma$, we find that three bodies of a co-circular central configuration cannot lie on the same half of the circumscribing circle as the longest side $r_{12}$ unless the configuration is a kite. 


\section{Conclusion}

We have used mutual distances as coordinates to fully classify the set of co-circular central configurations in the planar four-body problem. This set is a two-dimensional surface, a graph over the two shortest exterior side-lengths, whose boundaries are important symmetric families consisting of a kite, an isosceles trapezoid and an equilateral triangle with a zero mass off the triangle. A specific ordering of the masses has been demonstrated to hold for any co-circular c.c. Somewhat surprisingly, if just two masses of a co-circular c.c. are equal, then the configuration is symmetric, either a kite or an isosceles trapezoid. The set of positive masses which yield a co-circular c.c. lie in a two-dimensional algebraic variety. We conjecture that for those masses for which a co-circular c.c. exists, the configuration is unique.

By restricting the central configuration to lie on a circle, we have been able to utilize the resulting geometry as well as Ptolemy's theorem to obtain many results. It is our hope that this may spark similar efforts to use constraints in an attempt to classify general convex, four-body central configurations. For example, is it useful to search for c.c.'s under the constraint $P=c$, where $c \neq 0$ ? Are there other feasible geometric constraints whose level sets are tangent to $V=0$ ? Could these methods help find a foliation of the space of convex central configurations and suggest a technique for proving the uniqueness of such configurations? Finally, are any of the relative equilibria corresponding to the co-circular c.c.'s on $\Gamma$ linearly stable and if so, is it possible to rigorously prove this is the case? We hope to explore some of these interesting questions in future work.

Acknowledgment: Both authors would like to thank Dick Hall for bringing us together to work on this problem and for many helpful suggestions. We would also like to thank the referees for their constructive comments. Conversations with Marshall Hampton, Eduardo Leandro, John Little, Rick Moeckel, Manuele Santoprete and Deiter Schmidt during the time of this research were greatly appreciated. GR would like to thank the National Science Foundation (grant DMS-0708741) and the American Institute of Mathematics for their support. JC has been partially supported by the grants MEC/FEDER MTM2008-V03437 and CIRIT No. 2009SGR-410.

\section{References}

[1] Albouy, A., On a paper of Moeckel on central configurations, Regul. Chaotic Dyn. 8 (2003), no. $2,133-142$.

[2] Albouy, A. and Kaloshin, V., Finiteness of central configurations of five bodies in the plane, preprint, 2011.

[3] Albouy, A., Recherches sur le Problème des N Corps, Notes Scientifiques et Techniques du Bureau des Longitudes, 1997.

[4] Albouy, A., Fu, Y. and Sun, S., Symmetry of planar four-body convex central configurations, Proc. R. Soc. Lond. Ser. A 464 (2008), no. 2093, 1355-1365. 
[5] Apostol, T. M., Ptolemy's inequality and the chordal metric, Mathematics Magazine 40 (1967), no. 5, 233-235.

[6] Berger, M., Geometry I, Translated from French by M. Cole and S. Levy, Springer-Verlag, Berlin, 1987.

[7] Cox, D., Little, J. and O’Shea, D., Using Algebraic Geometry, 2nd. ed., Springer, New York, 2005.

[8] Dziobek, O., Über einen merkwürdigen Fall des Vielkörperproblems, Astro. Nach. 152 (1900), $32-46$.

[9] Fayçal, N., On the classification of pyramidal central configurations, Proc. Am. Math. Soc. 124 (1996), no. 1, 249-258.

[10] Hampton, M., Co-circular central configurations in the four-body problem, EQUADIFF 2003 (Conference Proceedings), World Sci. Publ., Hackensack, NJ, (2005), 993-998.

[11] Hampton, M., Concave central configurations in the four-body problem, Doctoral Thesis, Univ. of Washington, Seattle, 2002.

[12] Hampton, M. and Moeckel, R., Finiteness of relative equilibria of the four-body problem, Invent. Math. 163 (2006), no. 2, 289-312.

[13] Kulevich, J. L., Roberts, G. E. and Smith, C. J., Finiteness in the planar restricted four-body problem, Qual. Theory Dyn. Syst. 8 (2009), 357-370.

[14] Long, Y., Admissible shapes of 4-body non-collinear relative equilibria, Adv. Nonlinear Stud. 3 (2003), no. 4, 495-509.

[15] MacMillan, W. D. and Bartky, W., Permanent Configurations in the Problem of Four Bodies, Transactions of the American Mathematical Society 34 (1932), no. 4, 838-875.

[16] Maple, version 13.02 (2009), maplesoft, Waterloo Maple Inc.

[17] MATLAB, version 7.8.0.347 (2009), The MathWorks, Inc.

[18] Mello, L. F. and Fernandes, A. C., Co-circular and Co-spherical Kite Central Configurations, Qual. Theory Dyn. Syst. 10 (2011), 29-41.

[19] Meyer, K. R., Hall, G. R. and Offin, D., Introduction to Hamiltonian Dynamical Systems and the N-Body Problem, 2nd ed., Applied Mathematical Sciences, 90, Springer, New York, 2009.

[20] Moeckel, R., On central configurations, Mathematische Zeitschrift 205 (1990), no. 4, 499-517.

[21] Saari, D. G., Collisions, Rings, and Other Newtonian N-Body Problems, CBMS Regional Conference Series in Mathematics, no. 104, Amer. Math. Soc., Providence, RI, 2005.

[22] SAGE Mathematical Software, Version 3.1.1 (2008), http://www.sagemath.org 
[23] Santos, A. A., Alvarez-Ramírez, M., Vidal, C., On co-circular central configurations in the four and five-body problem for homogeneous force laws, preprint, 2011.

[24] Schmidt, D., Central configurations and relative equilibria for the $N$-body problem, Classical and celestial mechanics (Recife, 1993/1999), Princeton Univ. Press, Princeton, NJ, (2002), $1-33$.

[25] Schmidt, D., personal communication, June 19, 2010.

[26] Smale, S., Mathematical problems for the next century, Math. Intelligencer 20 (1998), no. 2, $7-15$.

[27] Sturmfels, B., Solving Systems of Polynomial Equations, CBMS Regional Conference Series in Mathematics, no. 97, Amer. Math. Soc., Providence, RI, 2002.

[28] Weisstein, E. W., Cyclic quadrilateral, from MathWorld-A Wolfram Web Resource, http://mathworld.wolfram.com/CyclicQuadrilateral.html.

[29] Wintner, A., The Analytical Foundations of Celestial Mechanics, Princeton Math. Series 5, Princeton University Press, Princeton, NJ, 1941.

[30] Xia, Z., Convex central configurations for the $n$-body problem, J. Differential Equations 200 (2004), 185-190. 